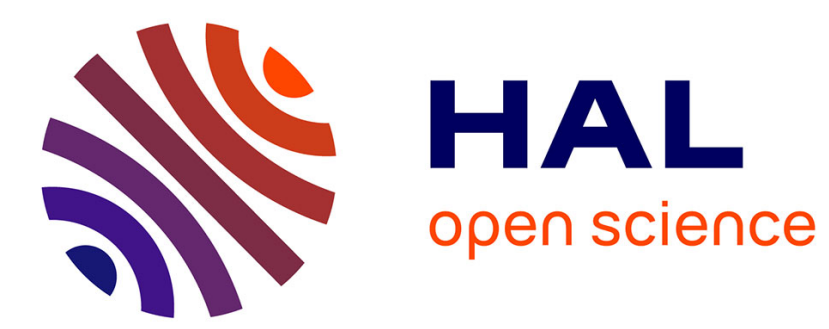

\title{
On long $\kappa$-tuples with few prime factors
}

Olivier Ramaré

\section{To cite this version:}

Olivier Ramaré. On long $\kappa$-tuples with few prime factors. Proceedings of the London Mathematical Society, 2012, 104 (1), pp.158-196. 10.1112/plms/pdr026 . hal-02572799

\section{HAL Id: hal-02572799 \\ https://hal.science/hal-02572799}

Submitted on 13 May 2020

HAL is a multi-disciplinary open access archive for the deposit and dissemination of scientific research documents, whether they are published or not. The documents may come from teaching and research institutions in France or abroad, or from public or private research centers.
L'archive ouverte pluridisciplinaire HAL, est destinée au dépôt et à la diffusion de documents scientifiques de niveau recherche, publiés ou non, émanant des établissements d'enseignement et de recherche français ou étrangers, des laboratoires publics ou privés. 


\title{
On long $\kappa$-tuples with few prime factors
}

\author{
O. Ramaré
}

\section{ABSTRACT}

We prove that there are infinitely many integers $n$ such that the total number of prime factors of $\left(n+h_{1}\right) \cdots\left(n+h_{\kappa}\right)$ is exactly $(1+o(1)) \kappa \log \kappa$. Our result even ensures us that these prime factors are fairly evenly distributed among every factors $n+h_{i}$.

\section{Introduction and some results}

The prime $\kappa$-tuple conjecture of Hardy \& Littlewood in [11] asserts that, given any "admissible" $\kappa$-tuple $\left(h_{1}=0, h_{2}, \cdots, h_{\kappa}\right)$, there are infinitely integers such that each of $n, n+$ $h_{2}, \cdots, n+h_{\kappa}$ is prime. By an admissible $\kappa$-tuple, we mean a $\kappa$-tuple $\left(h_{1}=0, h_{2}, \cdots, h_{\kappa}\right)$ of distinct non-negative integers such that the set $\left\{h_{1}, \cdots, h_{\kappa}\right\}$ does not cover all of $\mathbb{Z} / p \mathbb{Z}$, for every prime $p$. For instance, $(0,2)$ and $(0,2,6)$ are admissible tuples, while $(0,2,4)$ is not. This conjecture is of course out of reach of today's techniques, and the best one can do, since Brun in [4], is to produce tuples $\left(n, n+h_{2}, \cdots, n+h_{\kappa}\right)$ whose total number of prime factors is bounded in terms of $\kappa$. We are concerned in this paper with the case when $\kappa$ is large. The best available result is still the one of Miech from [16], where he proves that there exist infinitely many $\kappa$-tuples having in all at most $\kappa \log \kappa+\mathcal{O}(\kappa)$ prime factors. The best bound one can expect is $\kappa$ by the Hardy-Littlewood conjecture, but, more realistically and taking the parity barrier into account, the best bound we hope to achieve here is $2 \kappa$. As a matter of fact, the parity principle is the only identified hurdle here, but even getting a bound $\leq(1-\delta) \kappa \log \kappa$ for some positive $\delta$ has not been achieved. We will be confronted here to the same barrier, in a strong way, see the paragraph before Theorem 1.2, but will explain partly this phenomenom. The proof of the claim of [21, Proposition, page 287] is faulty, as far as I can see. Different bounds appear in [10, Theorem 10.5, 10.7, Corollary 10.11.2], [5, table 11.1]; all have the same asymptotic behaviour.

The main novelty in recent years is due to Heath-Brown in [12]. On developing an idea of Selberg on the twin prime conjecture, he investigated the problem of bounding the number of prime factors of each $n+h_{i}$ and has obtained that, given any admissible $\kappa$-tuple, there are infinitely many tuples $\left(n, n+h_{2}, \cdots, n+h_{\kappa}\right)$ such that each $n+h_{i}$ has at most $2(1+$ $o(1)) \log \kappa / \log 2$ prime factors.

Here is a typical corollary of the method we develop:

THEOREM 1.1. Given any admissible $\kappa$-tuple $\left(h_{1}, \cdots, h_{\kappa}\right)$ there are infinitely many integers $n$ such that

$$
(1 / \kappa) \sum_{1 \leq i \leq \kappa}\left(\omega\left(n+h_{i}\right)-\log \kappa\right)^{2} \ll \log \kappa
$$

where $\omega(m)$ denotes the number of prime factors of $m$ counted without multiplicity. 
In short, we are not only able to produce $\kappa$-tuples with at most $\kappa \log \kappa+\mathcal{O}(\kappa)$, but with exactly $\kappa \log \kappa+\mathcal{O}(\kappa \sqrt{\log \kappa})$. If we seek only this total number, we can also reach $\kappa \log \kappa+$ $\mathcal{O}(\kappa)$. Our result furthermore ensures that these primes factors are fairly evenly distributed among each factor $n+h_{i}$. As usual (with the exception of [7]), it is possible to ensure that there are more than $\gg N /(\log N)^{\kappa}$ such $\kappa$-tuples below $N$, and we can further guarantee them to be free of prime factors below $N^{\eta}$ for a small but positive $\eta$. We concentrate on the simpler situation, and on the novel aspects of the method we propose.

It is striking that the limit $\kappa \log \kappa$ should arise so vividly, and a partial explanation is this, anticipating somewhat on what follows: the host sequence $\beta(n)$ (see $(1.2)$ and sections 2 and 4) has in its support many integers that indeed are such that $\left(n+h_{1}\right) \cdots\left(n+h_{\kappa}\right)$ has $(1+o(1)) \kappa \log \kappa$ prime factors. Note that we are not able to produce integers in the support of $\beta(n)$ and such that $\left(n+h_{1}\right) \cdots\left(n+h_{\kappa}\right)$ has, say $(2+o(1)) \kappa \log \kappa$ prime factors. But it does not rule out their existence either.

The traditional way to study this problem introduces the sequence of numbers

$$
\Pi_{\left(h_{1}, \cdots, h_{\kappa}\right)}(n)=\prod_{1 \leq i \leq \kappa}\left(n+h_{i}\right)
$$

and looks for such numbers free of small prime factors. The so-called linearized approach restricts $n$ to primes and we can thus remove the factor $n+h_{1}=n$ from $\Pi_{\left(h_{1}, \cdots, h_{\kappa}\right)}(n)$. This is efficient when $\kappa$ is small, but we will neglect it since we are interested at what happens when $\kappa$ goes to infinity. Our study relies on the quantity

$$
S_{i}\left(\left(a_{d^{*}}\right)_{d^{*}}\right)=\sum_{n \leq N}\left(\sum_{d^{*} \mid n+h_{i}} a_{d^{*}}\right) \beta(n) \text { with } \beta(n)=\left(\sum_{d \mid \Pi_{\left(h_{1}, \cdots, h_{\kappa}\right)}(n)} \lambda_{d}\right)^{2} .
$$

This is a simple version of a more elaborate sum we will present later on, at (2.5). The coefficient $\beta(n)$ arises in this form in the Selberg sieve. Miech in [16] chooses precisely the one that is chosen there, while Salerno in [21] and Greaves in [8, Section 7.3.2], following ideas of [2] and of $[\mathbf{2 4}]$ both takes more elaborate versions, all explained thereafter.

The word "weight" being overloaded in this theory, we are in need of a stricter terminology. We call $\beta(n)$ the host sequence, while referring to the coefficient $c(n)=\sum_{d^{*} \mid n+h_{i}} a_{d^{*}}$ as the sieve coefficient. We will try to add $\mathrm{a}^{*}$ to what pertains to this coefficient. Two issues will make matters somewhat more intricate: the construction of the usual Selberg coefficients $\beta(n)$ depends on yet another sequence which, at this level, will be called (and be treated as) a host sequence. Secondly, we shall modify these Selberg coefficients by employing ... some weights! The word weight will be reserved for these, except in the expression the weighted sieve. The weighted sieve approach dealt with in this paper consists in showing that $\sum_{i} S_{i}\left(\left(a_{d^{*}}\right)_{d^{*}}\right)$ is non-negative for some sieve coefficients $c(n)$ and a given host sequence $\beta$.

The choice of a proper family of host sequences will be fairly satisfactorily answered to in this paper, but a proper choice of sieve coefficients remains hazy, though we will make some progress. Concerning this coefficient, the traditional choice takes it in the form

$$
c(n)=b-\sum_{\substack{p \leq P=N^{\theta}, p \mid \Pi_{\left(h_{1}, \cdots, h_{\kappa}\right)}(n)}} 1
$$

thus restricting the sum to $d^{*}$ being 1 or a prime number.

Historically speaking, the first weighted sieve had been used by Kuhn in 1941 [14] to prove that every interval $[X, X+\sqrt{X}]$ contains numbers having at most 4 prime factors, provided $X$ be large enough.

At this level, we skip the choice of the host sequence. It is detailed in section 2 and 4 . Each choice depends on a large parameter $Q$ and on a non-negative non-increasing function $w$ on $[0, \infty$ [ which furthermore is supposed to be continuous, piecewise differentiable with 
a bounded derivative and such that $w(1)=0$. This choice, or rather the way to handle the resulting coefficient, is the first novelty of this paper. The setting we create for them leads to easy treatments, so easy that we are in a position to handle sieve coefficients $c(n)$ that do not only depend on prime divisors of $\Pi_{\left(h_{1}, \cdots, h_{\kappa}\right)}(n)$ but also on divisors that have a given number of prime factors. We will examine the functions $w(t)=\max (0,1-t)^{\nu}$ for positive $\nu$ such that but $\log \nu=o\left(\log ^{2} \kappa\right)$, but we will attain the same bound $\kappa \log \kappa$, so giving somewhat more consistency to this barrier. We further consider a sequence: the characteristic function $a_{r}[P]$ of those integers that have exactly $r$ prime factors, all distinct and all $\leq P$, and 0 at any other ones.

THEOREM 1.2. For any non-negative integer $r$, any integer parameter $\kappa$ and any parameter $\tau>0$, there exists a bounded continuous function $\mathfrak{G}_{\kappa, r}\left(t_{1}, t_{2}, \tau\right)$ with the following property. Let $\left(h_{1}, \cdots, h_{\kappa}\right)$ be an admissible $\kappa$-tuple, let $Q \geq 1$ be a parameter and $w$ be a function as above. We consider the sum $S_{i}\left(a_{r}\left[Q^{\tau}\right]\right)$ from $(1.2)$ when $\beta(n)$ is as above. We have

$$
\begin{array}{r}
\frac{S_{i}\left(a_{r}\left[Q^{\tau}\right]\right)}{N /(\log Q)^{\kappa}}=\mathscr{C}\left(h_{1}, \cdots, h_{\kappa}\right) \int_{0 \leq t_{1}, t_{2} \leq 1} w^{\prime}\left(t_{1}\right) w^{\prime}\left(t_{2}\right) \mathfrak{G}_{\kappa, r}\left(t_{1}, t_{2}, \tau\right) d t_{1} d t_{2} \\
+\mathcal{O}\left(1 /(\log Q)^{1 / 9}\right)+\mathcal{O}\left(Q^{r \tau+2} N^{-1}(\log Q)^{\kappa}\right)
\end{array}
$$

where the constant $\mathscr{C}\left(h_{1}, \cdots, h_{\kappa}\right)$ is given by

$$
\mathscr{C}\left(h_{1}, \cdots, h_{\kappa}\right)=\prod_{p \geq 2}\left(1-\frac{\#\left\{h_{1}, \cdots, h_{\kappa} \bmod p\right\}}{p}\right)\left(1-\frac{1}{p}\right)^{-\kappa} .
$$

A more general Theorem in proved in Theorem 14.1. The expression we find for $\mathfrak{G}_{\kappa, r}\left(t_{1}, t_{2}, \tau\right)$ is fairly explicit but too complicated to get even the asymptotic dependence in $\kappa$. See $(17.1)$ and (18.1) in case $r=0$ and $r=1$. Let us summarize here the properties we prove:

(1) $\mathfrak{G}_{\kappa, r}$ is symetrical in $t_{1}$ and $t_{2}$, i.e. $\mathfrak{G}_{\kappa, r}\left(t_{1}, t_{2}, \tau\right)=\mathfrak{G}_{\kappa, r}\left(t_{2}, t_{1}, \tau\right)$.

(2) For any $\lambda>0$, we have $\mathfrak{G}_{\kappa, r}\left(t_{1}, t_{2}, \tau\right)=\lambda^{\kappa} \mathfrak{G}_{\kappa, r}\left(t_{1} / \lambda, t_{2} / \lambda, \tau / \lambda\right)$.

(3) $\mathfrak{G}_{\kappa, r}\left(t_{1}, 0, \tau\right)=\mathfrak{G}_{\kappa, r}\left(0, t_{2}, \tau\right)=0$ and we extend $\mathfrak{G}_{\kappa, r}\left(t_{1}, t_{2}, \tau\right)$ to negative values of $t_{1}$ and/or $t_{2}$ by attributing it the value 0 .

(4) $\mathfrak{G}_{\kappa, r}$ is a bounded continuous function.

(5) When $\tau>0$, the following recursion formula holds:

$$
\begin{aligned}
\tau \frac{d \mathfrak{G}_{\kappa, r}\left(t_{1}, t_{2}, \tau\right)}{d \tau}=\mathfrak{G}_{\kappa, r-1}\left(t_{1}, t_{2}, \tau\right)-\mathfrak{G}_{\kappa, r-1}\left(t_{1}-\tau, t_{2}, \tau\right) \\
\quad-\mathfrak{G}_{\kappa, r-1}\left(t_{1}, t_{2}-\tau, \tau\right)+\mathfrak{G}_{\kappa, r-1}\left(t_{1}-\tau, t_{2}-\tau, \tau\right) .
\end{aligned}
$$

(6) When $\tau>0$, we have $\mathfrak{G}_{\kappa, 0}\left(t_{1}, t_{2}, \tau\right)=\min \left(t_{1}, t_{2}\right)^{\kappa}$.

The formula (14.2) offers a distinct approach, as it avoids any notion of derivative, but we need to have access to the second derivative of $\mathfrak{G}_{\kappa, r}$ : we do not know how to prove that it exists in general. See (17.2) and (18.2) in case $r=0$ and $r=1$.

We will use sieve coefficients supported by products of two prime factors, in a general context; prove a precise formula for a specific host sequence for products of two primes, and prove a general but very cumbersome formula for general sieve coefficients supported by products of $r$ primes.

Final note: we restricted our attention in this work to prime $\kappa$-tuples and did not consider the case of a general $\kappa$-dimensional sieve setting, but similar results are available, at least when $\kappa$ is an integer. We also did not try to rule out primes appearing with multiplicities. In fact, we tried to stick to the simplest problem: the technique is still so heavy that we are not yet able to do all what one would wish, even in this special situation. 
Acknowledgements. We include in this paper a proof due to D.S. Ramana [18] of a Theorem that offers a control on the $L^{1}$ norm of the Fourier transform of a piecewise affine compactly supported function in several dimension. This Theorem is of independent interest and is included in section 11. I thank him warmly for this contribution.

The referee is also thanked for his/her careful reading of this paper.

Notation: We assume that $Q \geq 2$ and we set $\alpha=1 / \log Q, \tau=(\log P) /(\log Q)$ as well as $R=\log P_{0}$ where $P_{0} \leq P$ will be introduced later. We also set $\varepsilon=\alpha R$ and $Z=(\log Q)^{\kappa}$. We use

$$
\mathcal{H}_{m}=\sum_{1 \leq k \leq m} 1 / k .
$$

The symbol + as an exponent will be used to avoid negative values: $x^{+}=\max (0, x)$ and $x^{+m}=\max (0, x)^{m}$. We close this part by a remark concerning one-dimensional integrals. We use most often $\int_{a \leq t \leq b} f(t) d t$ and not $\int_{a}^{b} f(t) d t$ : the first one vanishes when $b<a$ while, in such a situation, the second one is commonly understood as $-\int_{b}^{a} f(t) d t$.

\section{The approach in the large}

For an admissible shift $\left(h_{1}, \cdots, h_{\kappa}\right)$, we define

$$
\mathcal{K}\left(h_{1}, \cdots, h_{\kappa}\right)_{d}=\bigcap_{1 \leq i \leq \kappa}\left(\mathcal{U}_{d}-h_{i}\right) \quad\left(\text { with } \mathcal{U}_{d}=(\mathbb{Z} / d \mathbb{Z})^{*}\right)
$$

When $n$ lies in $\mathcal{K}\left(h_{1}, \cdots, h_{\kappa}\right)_{d}$, then $n+h_{i}$ falls in $\mathcal{U}_{d}$, i.e. is prime to $d$, for all $i$ from 1 to $\kappa$.

We proposed some time ago (see $[\mathbf{2 0}]$ and $[\mathbf{1 9}]$ ) a geometrical approach that dispenses with building an auxiliary polynomial, like $\Pi_{\left(h_{1}, \cdots, h_{\kappa}\right)}$ above. The exposition will be made easier by the following definition.

Definition 2.1. A collection $\left(\mathcal{K}_{d}\right)_{d \geq 1}$ is said to be a multiplicatively split compact set when

(i) For each $d \geq 1, \mathcal{K}_{d}$ is a subset of $\mathbb{Z} / d \mathbb{Z}$.

(ii) When $d_{1}$ and $d_{2}$ are co-prime, $\mathcal{K}_{d_{1} d_{2}}$ is in one to one correspondance, via the Chinese remainder map, with $\mathcal{K}_{d_{1}} \times \mathcal{K}_{d_{2}}$.

We further say that it is square-free when

(iii) If $d_{1} \mid d_{2}$ but both have the same prime factors, $\mathcal{K}_{d_{2}}$ is the reverse image of $\mathcal{K}_{d_{1}}$ through the canonical surjection from $\mathbb{Z} / d_{2} \mathbb{Z}$ to $\mathbb{Z} / d_{1} \mathbb{Z}$.

The main compact set we will consider is $\mathcal{K}\left(h_{1}, \cdots, h_{\kappa}\right)$. Its definition $(2.1)$ shows clearly that it is multiplicatively split and square-free. We need another one, $\mathcal{K}^{*}$, that will be successively $\mathcal{U}_{d}-h_{1}, \mathcal{U}_{d}-h_{2}, \ldots, \mathcal{U}_{d}-h_{\kappa}$. In general we simply assume that

$\left(H_{1}\right) \mathcal{K}$ is a multiplicatively split compact set;

$\left(H_{2}\right) \mathcal{K}^{*}$ is a square-free multiplicatively split compact set that contains $\mathcal{K}$.

We associate to $\mathcal{K}^{*}$ (and to any multiplicatively split compact set) its bordering system $\left(\mathcal{L}_{d}^{*}\right)_{d \geq 1}$. Each $\mathcal{L}_{d}^{*}$ is the subset of $\mathbb{Z} / d \mathbb{Z}$ defined by

$-\mathcal{L}_{1}^{*}=\{1\}$ and $\mathcal{L}_{d}^{*}=\emptyset$ when $d$ is not square-free.

- When $d_{1}$ and $d_{2}$ are co-prime, $\mathcal{L}_{d_{1} d_{2}}^{*}$ is in bijection via the Chinese remainder map to $\mathcal{L}_{d_{1}}^{*} \times \mathcal{L}_{d_{2}}^{*}$.

- $\mathcal{L}_{p}^{*}=\mathbb{Z} / p \mathbb{Z} \backslash \mathcal{K}_{p}^{*}$. 
This may look complicated, but the situation clears when one looks at characteristic functions (the expressions are valid whether the compact set $\mathcal{K}^{*}$ be square-free or not):

$$
\mathbb{1}_{\mathcal{L}_{d}^{*}}=\prod_{p^{\nu} \| d}\left(\mathbb{1}_{\mathcal{K}_{p^{\nu-1}}}-\mathbb{1}_{\mathcal{K}_{p^{\nu}}}\right)=(-1)^{\omega(d)} \sum_{\delta \mid d} \mu(d / \delta) \mathbb{1}_{\mathcal{K}_{\delta}}
$$

and

$$
\mathbb{1}_{\mathcal{K}_{d}}=\prod_{p^{\nu} \| d}\left(\mathbb{1}-\mathbb{1}_{\mathcal{L}_{p}^{*}}-\cdots-\mathbb{1}_{\mathcal{L}_{p^{\nu}}^{*}}\right)=\sum_{\delta \mid d}(-1)^{\omega(\delta)} \mathbb{1}_{\mathcal{L}_{\delta}^{*}} .
$$

We will also use the bordering system $\left(\mathcal{L}_{d}\right)_{d \geq 1}$ associated with $\mathcal{K}$. Note that the condition $\mathcal{K}^{*} \supset \mathcal{K}$ has the following consequence: when $p$ is a prime number and $a$ is a positive integer, we have

$$
\mathbb{1}_{\mathcal{K}_{p^{a}}} \cdot \mathbb{1}_{\mathcal{L}_{p}^{*}}=\mathbb{1}_{\mathcal{K}_{p^{a}}} \cdot\left(\mathbb{1}-\mathbb{1}_{\mathcal{K}_{p}^{*}}\right)=0 .
$$

Having this preparation at hand, we can present the main actor of this paper, namely the sum

$$
S\left(\left(a_{d^{*}}\right)_{d^{*}} ; \mathcal{K}, \mathcal{K}^{*}\right)=\sum_{n \leq N}\left(\sum_{d^{*} / n \in \mathcal{L}_{d^{*}}^{*}} a_{d^{*}}\right)\left(\sum_{d / n \in \mathcal{K}_{d}} \lambda_{d}^{\sharp}\right)^{2} .
$$

We assume that $a_{d^{*}}$ vanishes when $d^{*}>D^{*}$. The coefficients $\left(\lambda_{d}^{\sharp}\right)_{d}$ are completely free for us to choose. We simply assume that they vanish when $d>Q$, for some parameter $Q$. We need some more material from sieve theory. We define the coefficients $\left(\lambda_{d}\right)_{d}$ by

$$
\lambda_{d}=(-1)^{\omega(d)} \sum_{d \mid \ell} \lambda_{\ell}^{\sharp}
$$

We have (see $[\mathbf{1 9},(11.5)])$

$$
\lambda_{d}^{\sharp}=\sum_{d \mid \ell} \mu(\ell / d)(-1)^{\omega(\ell)} \lambda_{\ell} \quad \text { and } \sum_{d / n \in \mathcal{K}_{d}} \lambda_{d}^{\sharp}=\sum_{d / n \in \mathcal{L}_{d}} \lambda_{d} .
$$

In practice, the condition $n \in \mathcal{K}_{d}$ leads to easier treatment of the main term, while the $\lambda_{d}$ 's will be smaller, leading to a better treatment of the error term. We finally introduce the multiplicative function

$$
h(d)=\prod_{p^{\nu} \| d}\left(\frac{p^{\nu}}{\left|\mathcal{K}_{p^{\nu}}\right|}-\frac{p^{\nu-1}}{\left|\mathcal{K}_{p^{\nu-1}}\right|}\right) .
$$

We need to handle averages of this function and we follow [10, Chapter 5] (see also [9]). Condition $\left(\Omega_{1}\right)$ therein is introduced in the fourth part of the first chapter, page 49 , but it is expedient to assume a much stronger hypothesis, namely

$$
h(p) \ll \kappa / p .
$$

Our main hypothesis on $h$ is a slight simplification of what is called $\left(\Omega_{2}(\kappa, L)\right)$ at the beginning of $[\mathbf{1 0}$, Chapter 5]. It reads

$$
\sum_{p \leq x} \frac{h(p) \log p}{p}=\kappa \log x+\mathcal{O}(1) .
$$

Note that we will use the fact that $\kappa$ is an integer in section 12 . This implies classically that

$$
\sum_{\delta \leq x} h(\delta)=A(\log x)^{\kappa}+\mathcal{O}\left((\log (2 x))^{\kappa-1}\right)
$$

when $x \leq Q$, by using [10, Lemma 5.3, 5.4] and Merten's Theorem. We deduce from $\left(H_{3}\right)$ and $\left(H_{4}\right)$ the following weaker form which will be easier to use:

$$
\sum_{\delta \leq x} h(\delta)=A(\log x)^{\kappa}+\mathcal{O}(\alpha Z) . \quad(1 \leq x \leq Q) .
$$


When $\mathcal{K}=\mathcal{K}\left(h_{1}, \cdots, h_{\kappa}\right)$, the constant $A$ is equal to the constant $\mathscr{C}$ of Theorem 1.2 . It is $>0$ when $\left(h_{1}, \cdots, h_{\kappa}\right)$ is admissible, or, and this is an equivalent statement, when $\mathcal{K}\left(h_{1}, \cdots, h_{\kappa}\right)$ is non-empty.

\section{Generalisation of a formula of Bombieri}

The quantity (2.5) has a summation over four variables $\left(n, d^{*}, d\right.$ and $\left.d^{\prime}\right)$. We take care here of the summation over $n$. We set

$$
\gamma\left(d^{*}\right)=\left|\mathcal{L}_{d^{*}}^{*}\right| / d^{*}
$$

as well as

$$
S_{0}\left(\left(a_{d^{*}}\right)_{d^{*}} ; \mathcal{K}, \mathcal{K}^{*}\right)=\sum_{d^{*}, \delta} \gamma\left(d^{*}\right) a_{d^{*}} h(\delta)\left(\sum_{\substack{\delta \mid d,\left(d, d^{*}\right)=1}}\left|\mathcal{K}_{d}\right| \lambda_{d}^{\sharp} / d\right)^{2}
$$

LEMMA 3.1. We have

$$
S\left(\left(a_{d^{*}}\right)_{d^{*}} ; \mathcal{K}, \mathcal{K}^{*}\right)=N S_{0}\left(\left(a_{d^{*}}\right)_{d^{*}} ; \mathcal{K}, \mathcal{K}^{*}\right)
$$

$$
+\mathcal{O}\left(\sum_{d_{1}^{*}, d_{2}, d_{3}}\left|a_{d_{1}^{*}}\right|\left|\lambda_{d_{2}}\right|\left|\lambda_{d_{3}}\right|\left|\mathcal{L}_{d_{1}}^{*}\right|\left|\mathcal{L}_{d_{2}}\right|\left|\mathcal{L}_{d_{3}}\right|\right)
$$

Proof. We first revert to $\left(\mathcal{L}_{d}\right)$ on invoking $(2.7)$ and get

$$
S\left(\left(a_{d^{*}}\right)_{d^{*}} ; \mathcal{K}, \mathcal{K}^{*}\right)=\sum_{d_{1}^{*}, d_{2}, d_{3}} a_{d_{1}^{*}} \lambda_{d_{2}} \lambda_{d_{3}} \sum_{\substack{n \leq N, n \in \mathcal{L}_{d_{1}^{*}}^{*} \cap \mathcal{L}_{d_{2}} \cap \mathcal{L}_{d_{3}}}} 1 .
$$

Note that $\mathcal{L}_{d_{1}^{*}}^{*} \cap \mathcal{L}_{d_{2}} \cap \mathcal{L}_{d_{3}}$ vanishes when $d_{1}^{*}$ is not square-free, or when there is a prime $p$ and two distinct powers $a \geq 1$ and $b \geq 1$ that divides respectively $d_{2}$ and $d_{3}$. The reader will conclude that this set defines modulo $\left[d_{1}^{*}, d_{2}, d_{3}\right]$ a subset of cardinality at most $\left|\mathcal{L}_{d_{1}}^{*}\right|\left|\mathcal{L}_{d_{2}}\right|\left|\mathcal{L}_{d_{3}}\right|$. Concerning the main term, we divide it by $N$ and write it as

$$
\mathrm{M}=\sum_{d_{1}^{*}, d_{2}, d_{3}} a_{d_{1}^{*}} \lambda_{d_{2}} \lambda_{d_{3}} \frac{\left|\mathcal{L}_{d_{1}^{*}}^{*} \cap \mathcal{L}_{d_{2}} \cap \mathcal{L}_{d_{3}}\right|}{\left[d_{1}^{*}, d_{2}, d_{3}\right]} .
$$

It can be defined as the limit when $N$ goes to infinity of $S\left(\left(a_{d^{*}}\right)_{d^{*}} ; \mathcal{K}, \mathcal{K}^{*}\right) / N$. To compute this limit we use (2.7) and switch to the $\lambda_{d}^{\sharp}$. This gives us

$$
\mathrm{M}=\sum_{d_{1}^{*}, d_{2}, d_{3}} a_{d_{1}^{*}} \lambda_{d_{2}}^{\sharp} \lambda_{d_{3}}^{\sharp} \frac{\left|\mathcal{L}_{d_{1}^{*}}^{*} \cap \mathcal{K}_{d_{2}} \cap \mathcal{K}_{d_{3}}\right|}{\left[d_{1}^{*}, d_{2}, d_{3}\right]} .
$$

Note that $\mathcal{K}_{d_{2}} \cap \mathcal{K}_{d_{3}}=\mathcal{K}_{\left[d_{2}, d_{3}\right]}$. We use equation (2.4) to introduce the condition $\left(d_{1}^{*}, d_{2} d_{3}\right)=1$. This gives us

$$
\frac{\left|\mathcal{L}_{d_{1}^{*}}^{*} \cap \mathcal{K}_{d_{2}} \cap \mathcal{K}_{d_{3}}\right|}{\left[d_{1}^{*}, d_{2}, d_{3}\right]}=\frac{\left|\mathcal{L}_{d_{1}^{*}}^{*}\right|}{d_{1}^{*}} \frac{\left|\mathcal{K}_{d_{2}} \cap \mathcal{K}_{d_{3}}\right|}{\left[d_{2}, d_{3}\right]} .
$$

We complete the separation of $d_{2}$ and $d_{3}$ via the diagonalisation process of Selberg, i.e. we write

$$
\frac{\left|\mathcal{K}_{d_{2}} \cap \mathcal{K}_{d_{3}}\right|}{\left[d_{2}, d_{3}\right]}=\frac{\left|\mathcal{K}_{d_{2}}\right|\left|\mathcal{K}_{d_{3}}\right|}{d_{2} d_{3}} \sum_{\substack{\delta\left|d_{1} \\ \delta\right| d_{2}}} h(\delta)
$$


The Lemma follows readily.

This Lemma generalizes [2, Theorem 18]. This same formula occurs as [8, Section 7.3.1, Lemma 1]. This is also [24, (5.6')]. Our proof is much shorter than the initial one of Bombieri. Greaves's proof is also remarkably short and shares with the above one the fact of treating the variable $d_{1}^{*}$ in a distinct manner. Our switching between $\mathcal{L}$ and $\mathcal{K}$ as usual enables us to extend the proof to the case when $\mathcal{K}$ is not assumed to be square-free.

\section{A family of host sequences}

It is time to narrow our family of host sequences. But to do so, we will first develop some material to motivate our choice. Let $M=\operatorname{lcm}(d \leq Q)$ and let us look at $\mathcal{K}_{M} \subset \mathbb{Z} / M \mathbb{Z}$. We assume momentarily that the compact set satisfies the Johnsen-Gallagher condition (see [6], $[\mathbf{2 3}],[\mathbf{2 0}],[\mathbf{1 9}])$, i.e.

$$
\forall d \leq Q, \forall d \mid q, \forall a \in \mathcal{K}_{d}
$$

the number $\left|\left\{b \in \mathcal{K}_{q} / b \equiv a[d]\right\}\right|$ is independent of $a$.

Let us expand the characteristic function of $\mathcal{K}_{M}$ in Fourier series:

$$
\begin{aligned}
\mathbb{1}_{\mathcal{K}_{M}}(n) & =\sum_{b}\left(\frac{1}{M} \sum_{c \in \mathcal{K}_{M}} e(-b c / M)\right) e(b n / M) \\
& =\sum_{d \mid M a} \sum_{\bmod *_{d} d}\left(\frac{1}{M} \sum_{c \in \mathcal{K}_{M}} e(-a c / d)\right) e(a n / d) \\
& =\sum_{d \mid M a} \sum_{\bmod ^{*} d}\left(\frac{\left|\mathcal{K}_{M}\right|}{M\left|\mathcal{K}_{d}\right|} \sum_{c \in \mathcal{K}_{d}} e(-a c / d)\right) e(a n / d)
\end{aligned}
$$

where we have used the Johnsen-Gallagher condition (see (JG)). We define

$$
\psi_{d}^{*}(n)=\sum_{a}\left(\frac{1}{\left|\mathcal{K}_{d}\right|} \sum_{c \in \mathcal{K}_{d}} e(-a c / d)\right) e(a n / d) .
$$

These functions are, up to a multiplicative factor, the pseudo-characters introduced by Selberg in 1973 (see [2], [17] as well as [13]). An $\mathrm{L}^{2}$ approximation of $\mathbb{1}_{\mathcal{K}_{M}}$ is thus given by

$$
\frac{\left|\mathcal{K}_{M}\right|}{M} \sum_{d \leq Q} \psi_{d}^{*}(n)
$$

It is also possible to define $\psi_{d}^{*}(n)$ by Moebius inversion since we readily verify that

$$
\sum_{d \mid q} \psi_{d}^{*}=\frac{q}{\left|\mathcal{K}_{q}\right|} \mathbb{1}_{\mathcal{K}_{q}} .
$$

The coefficient $q /\left|\mathcal{K}_{q}\right|$ is somewhat mysterious and explained in [19, Section 9.4]. Inverting the above equation leads to the definition

$$
\psi_{d}^{*}=\sum_{q \mid d} \mu(d / q) \frac{q}{\left|\mathcal{K}_{q}\right|} \mathbb{1}_{\mathcal{K}_{q}} .
$$

This definition is valid whether $\mathcal{K}$ verifies condition (JG) or not, and is thus the one we take in general. Note that $\psi_{d}^{*}(n)=h(d)$ as soon as $n$ belongs to $\mathcal{K}_{d}$. As a consequence, the function

$$
\sum_{d \leq Q} \psi_{d}^{*}
$$


is constant over $\mathcal{K}_{M}$. Squared, this is the usual Selberg coefficient up to a normalising coefficient. The expression above calls immediately for a modification, namely

$$
\sum_{d \leq Q} \zeta_{d} \psi_{d}^{*}
$$

for some arbitrary coefficients $\left(\zeta_{d}\right)_{d \leq Q}$. We readily find that

$$
\begin{aligned}
\sum_{d \leq Q} \zeta_{d} \psi_{d}^{*}(n) & =\sum_{d \leq Q} \zeta_{d} \sum_{\substack{q \mid d, n \in \mathcal{K}_{q}}} \mu(d / q) \frac{q}{\left|\mathcal{K}_{q}\right|} \\
& =\sum_{q / n \in \mathcal{K}_{q}} \frac{q}{\left|\mathcal{K}_{q}\right|} \sum_{q \mid d \leq Q} \zeta_{d} \mu(d / q)
\end{aligned}
$$

so that we take

$$
\lambda_{q}^{\sharp}=\frac{q}{\left|\mathcal{K}_{q}\right|} \sum_{q \mid d \leq Q} \zeta_{d} \mu(d / q) / Z
$$

where $Z$ is a size parameter. Note that, when $d^{*}$ is square-free and co-prime with $\delta$,

$$
Z \sum_{\substack{\delta \mid d,\left(d, d^{*}\right)=1}}\left|\mathcal{K}_{d}\right| \lambda_{d}^{\sharp} / d=\sum_{\substack{\delta \mid d,\left(d, d^{*}\right)=1}} \sum_{d \mid q \leq Q} \zeta_{q} \mu(q / d)=\sum_{\delta \mid q} \zeta_{q} \sum_{\begin{array}{c}
\delta|d| q, \\
\left(d, d^{*}\right)=1
\end{array}} \mu(q / d) .
$$

Let us write $q=\delta \ell$. It is obvious that $\ell$ is prime to $d^{*}$. Furthermore

$$
\sum_{\substack{\delta|d| \delta \ell,\left(d, d^{*}\right)=1}} \mu(\delta \ell / d)=\mu(\ell)
$$

(since only $d=\delta$ appears in this sum) which does not vanish only when $\mu^{2}(\ell) \neq 0$, i.e. $\ell \mid d^{*}$. We define

$$
G_{d}(Q)=\sum_{\substack{f \leq Q,[f, d] \leq Q}} h(f) \zeta_{[d, f]} .
$$

This leads to

$$
\lambda_{d}=(-1)^{\omega(d)} G_{d}(Q) / Z
$$

The usual normalisation $Z=G_{1}(Q)$ is not required here because all our quantities are homogeneous with respect to $Z$. The next Lemma in a generalisation of $[\mathbf{2 6}]$ :

LEMma 4.1. When $\zeta \geq 0$ decreases on chains of multiples, the inequalities $G_{\ell}(Q \ell / d) \leq$ $G_{d}(Q) \leq G_{\ell}(Q)$ hold whenever $\ell \mid d$.

This has the nice consequence that $\left|\lambda_{d}\right| \leq G_{1}(Q) / Z$, while $\lambda_{d}^{\sharp}$ can be much bigger.

Proof: The condition $[f, d] \leq Q$ implies that $[f, \ell] \leq Q$, which proves the first claim (notice that $h \geq 0)$. In the other direction, let $f$ be such that $[f, \ell] \leq Q$. We have $[f, d] \leq[f, \ell](d / \ell)$ and the Lemma follows readily.

With such a choice of the $\lambda_{d}$ 's, $S_{0}\left(\left(a_{d^{*}}\right)_{d^{*}} ; \mathcal{K}, \mathcal{K}^{*}\right)$ becomes

$$
Z^{2} S_{0}\left(\left(a_{d^{*}}\right)_{d^{*}} ; \mathcal{K}, \mathcal{K}^{*}\right)=\sum_{\substack{d^{*}, \delta,\left(d^{*}, \delta\right)=1}} \gamma\left(d^{*}\right) a_{d^{*}} h(\delta)\left(\sum_{\substack{\ell \leq Q / \delta, \ell \mid d^{*}}} \mu(\ell) \zeta_{\delta \ell}\right)^{2}
$$


To compare with earlier work, our family of parameters $\left(\zeta_{d}\right)$ has this name in $[\mathbf{2}]$ and $[\mathbf{2 1}]$. Salerno in $[\mathbf{2 1}]$ chooses for $\zeta_{d}$ a step function with only two steps. Greaves in [8, Section 7.3.2, $(2.4)]$ calls this parameter $y(d)$ and chooses a logarithmic smoothing of $\mathbb{1}_{d \leq Q}$. Selberg in $[\mathbf{2 4}$, (7.6), (7.9), (7.11)] uses weights that are similar to (4.11). The paper [7, (3.14)] uses also a similar shape though the sum they study is somewhat different. Heath-Brown in $[\mathbf{1 2},(4)]$ determines $\lambda_{d}$ directly, but the sum he studies differs notably from ours.

We shall further restrict our attention to weights of shape

$$
\zeta_{d}=w\left(\frac{\log d}{\log Q}\right)=w(\alpha \log d)
$$

for some non-negative non-increasing function $w$ on $(0,1]$. We further assume that $w$ is continuous with $w(1)=0$, prolonged to $(0, \infty)$ by setting $w(t)=0$ when $t \geq 1$, piecewise differentiable and such that $w^{\prime}$ is bounded. These hypothesis ensure that $w(t)=-\int_{t}^{1} w^{\prime}(u) d u$ which is what we need. We thus have

$$
\zeta_{d}=-\int_{\alpha \log d}^{1} w^{\prime}(u) d u .
$$

\section{Two reduction steps}

In this section, we first restrict $d^{*}$ to integers without any small prime factors. This step may seem harmless and usual, but is in fact crucial; it will rid us of many constant terms in asymptotic expressions and will enable us to disregard most of the coprimality conditions. This introduces a parameter $P_{0}$ (and later $\left.\varepsilon=\left(\log P_{0}\right) / \log Q\right)$ which should disappear from the main term. However showing that the limit of this main term as $\varepsilon$ goes to zero exists is more difficult than expected and will be the subject of the technical sections 12 and 13 .

To restrict $d^{*}$, we limit our investigation to integers with fairly few divisors, as quantified by $\left(H_{5}\right)$ below.

Our second step here will be to remove the coprimality condition $\left(d^{*}, \delta\right)=1$ from (4.10).

Lemma 5.1. Let $d^{*}>1$ be an integer and $p$ be its smallest prime factor. We have, with the choice given by (4.12),

$$
\left|\sum_{\substack{\ell^{\prime} \leq Q / \delta, \ell^{\prime} \mid d^{*}}} \mu\left(\ell^{\prime}\right) \zeta_{\delta \ell^{\prime}}\right| \leq \tau\left(d^{*}\right)\left\|w^{\prime}\right\|_{\infty} \alpha \log p .
$$

Proof: Indeed, it is enough to consider the case when $d^{*}$ is square-free. Let us set $d^{*}=p d_{0}^{*}$. We can dispense with the condition $\ell^{\prime} \leq Q / \delta$ since it is included in $w$ (for $w(1)=0$ ). We thus find that

$$
\sum_{\substack{\ell^{\prime} \leq Q / \delta, \ell^{\prime} \mid d^{*}}} \mu\left(\ell^{\prime}\right) \zeta_{\delta \ell^{\prime}}=\sum_{\ell^{\prime} \mid d_{0}^{*}} \mu\left(\ell^{\prime}\right)\left(\zeta_{\delta \ell^{\prime}}-\zeta_{\delta p \ell^{\prime}}\right)
$$

which gets majorized as announced.

We assume that

$$
\sum_{d^{*}} \tau\left(d^{*}\right)^{2} \gamma\left(d^{*}\right)\left|a_{d^{*}}\right|=o(\log Q)
$$

This hypothesis follows from $\left(H_{7}\right)$ below for the sequence $a_{r}[P]$, the upper bound being $\mathcal{O}\left((\log \log P)^{r}\right)$. 
Removing the small prime factors of $d^{*} \quad$ Getting rid of the small prime factors of $d^{*}$ will simplify the computation of the main term, essentially by removing constant terms. Let $P_{0}$ be a parameter to be chosen later. We set

$$
\mathfrak{f}_{0}=\prod_{p \leq P_{0}} p
$$

We find that, on invoking $\left(H_{5}\right)$,

$$
\begin{aligned}
& \left|\sum_{\substack{\delta,\left(d^{*}, f_{0}\right) \neq 1}} \gamma\left(d^{*}\right) h(\delta) a_{d^{*}}\left(\sum_{\substack{\ell^{\prime} \leq Q / \delta, \ell^{\prime} \mid d^{*}}} \mu\left(\ell^{\prime}\right) \zeta_{\delta \ell^{\prime}}\right)^{2}\right| \\
& \quad \leq \alpha^{2}\left\|w^{\prime}\right\|_{\infty}^{2} Z\left(\log P_{0}\right)^{2} \sum_{d^{*}} \tau\left(d^{*}\right)^{2} \gamma\left(d^{*}\right)\left|a_{d^{*}}\right| \ll\left\|w^{\prime}\right\|_{\infty}^{2} \alpha Z\left(\log P_{0}\right)^{2} .
\end{aligned}
$$

This is more than enough. It is also $\mathcal{O}\left(\left\|w^{\prime}\right\|_{\infty}^{2} Z(\log \log P)^{r}\left(\log P_{0}\right)^{2}\right)$ for the sequence $a_{r}[P]$ under $\left(H_{7}\right)$.

Removing the coprimality condition We now remove the condition $\left(d^{*}, \delta\right)=1$ in $(4.10)$. Indeed on using $\left(H_{4}\right)$, we find that

$$
\begin{aligned}
& \left|\sum_{\begin{array}{r}
d^{*}, \delta, \\
\left(d^{*}, f_{0}\right)=1, \\
\left(d^{*}, \delta\right) \neq 1
\end{array}} \gamma\left(d^{*}\right) h(\delta) a_{d^{*}}\left(\sum_{\substack{\ell^{\prime} \leq Q / \delta,\left(d^{*}, f_{0}\right)=1, \ell^{\prime} \mid d^{*}}} \mu\left(\ell^{\prime}\right) \zeta_{\delta \ell^{\prime}}\right)^{2}\right| \\
& \leq \alpha^{2}\left\|w^{\prime}\right\|_{\infty}^{2} \sum_{P_{0}<p \leq P} \log ^{2} p \sum_{\substack{d^{*}, \delta,\left(d^{*}, f_{0}\right)=1, p\left|d^{*}, p\right| \delta}} \gamma\left(d^{*}\right) h(\delta)\left|a_{d^{*}}\right| \tau\left(d^{*}\right)^{2} \\
& \ll \alpha^{2}\left\|w^{\prime}\right\|_{\infty}^{2} \sum_{P_{0}<p \leq P} \frac{\log ^{2} p}{p} \sum_{\substack{d^{*}, \delta,\left(d^{*}, f_{0}\right)=1, p\left|d^{*}, p\right| \delta}} \gamma\left(d^{*}\right) h(\delta / p)\left|a_{d^{*}}\right| \tau\left(d^{*}\right)^{2} \\
& \ll \alpha^{2}\left\|w^{\prime}\right\|_{\infty}^{2} Z \sum_{\substack{d^{*} \\
\left(d^{*}, \mathfrak{f}_{0}\right)=1}} \gamma\left(d^{*}\right)\left|a_{d^{*}}\right| \tau\left(d^{*}\right)^{2} \sum_{p \mid d^{*}} \frac{\log ^{2} p}{p} .
\end{aligned}
$$

Note that $d^{*}$ has at most $\left(\log D^{*}\right) / \log P_{0}$ prime factors and that we have assumed that $\alpha \log D^{*} \ll 1$. As a consequence, the bound above is

$$
\ll\left\|w^{\prime}\right\|_{\infty}^{2} Z^{2} \alpha\left(\log P_{0}\right) / P_{0} .
$$

We set

$$
Z^{2} S_{0}^{(1)}\left(\left(a_{d^{*}}\right)_{d^{*} \leq D^{*}}\right)=\sum_{\substack{d^{*}, \delta,\left(d^{*}, f_{0}\right)=1}} \gamma\left(d^{*}\right) h(\delta) a_{d^{*}}\left(\sum_{\substack{\ell^{\prime} \leq Q / \delta, \ell^{\prime} \mid d^{*}}} \mu\left(\ell^{\prime}\right) \zeta_{\delta \ell^{\prime}}\right)^{2}
$$

and we can replace $S_{0}\left(\left(a_{d^{*}}\right)_{d^{*} \leq D^{*}}\right)$ by $S_{0}^{(1)}\left(\left(a_{d^{*}}\right)_{d^{*} \leq D^{*}}\right)$ up to an error term of size at most (up to a multiplicative constant)

$$
\left\|w^{\prime}\right\|_{\infty}^{2} Z \alpha\left(\left(\log P_{0}\right)^{2}+\left(\log P_{0}\right) / P_{0}\right) \ll\left\|w^{\prime}\right\|_{\infty}^{2} Z \alpha\left(\log P_{0}\right)^{2} .
$$




\section{Summing over $\delta$}

We open the square in (5.2) to get

$$
\begin{aligned}
& Z^{2} S_{0}^{(1)}\left(\left(a_{d^{*}}\right)_{d^{*} \leq D^{*}}\right)=\sum_{\substack{d^{*},\left(d^{*}, \mathfrak{f}_{0}\right)=1}} \gamma\left(d^{*}\right) a_{d^{*}} \sum_{\ell_{1}, \ell_{2} \mid d^{*}} \mu\left(\ell_{1}\right) \mu\left(\ell_{2}\right) \sum_{\delta} h(\delta) \zeta_{\ell_{1} \delta} \zeta_{\ell_{2} \delta}
\end{aligned}
$$

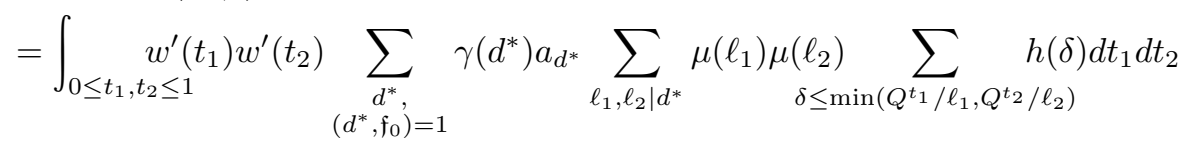

on invoking (4.12). We appeal to (2.8) at this level. This leads to an error term which is:

$$
\ll \alpha Z\|w\|_{1}^{2} \sum_{\substack{d^{*},\left(d^{*}, f_{0}\right)=1}} \gamma\left(d^{*}\right) \tau\left(d^{*}\right)^{2}\left|a_{d^{*}}\right|=o\left(\left\|w^{\prime}\right\|_{1}^{2} Z\right)
$$

by $\left(H_{5}\right)$. We have thus approximated $Z^{2} S_{0}^{(1)}\left(\left(a_{d^{*}}\right)_{d^{*} \leq D^{*}}\right)$ up to an error term of size $o\left(\left\|w^{\prime}\right\|_{1}^{2} Z\right)$ by

$$
\begin{aligned}
& Z^{2} S_{0}^{(2)}\left(\left(a_{d^{*}}\right) d^{*} \leq D^{*}\right) \\
& =A \sum_{\substack{\ell_{1}, \ell_{2} \leq D^{*},\left(\ell_{1} \ell_{2}, \mathfrak{f}_{0}\right)=1}} \mu\left(\ell_{1}\right) \mu\left(\ell_{2}\right) \Theta\left(\ell_{1}, \ell_{2}\right) \sum_{\substack{d^{*},\left(d^{*}, f_{0}\right)=1,\left[\ell_{1}, \ell_{2}\right] d^{*}}} \gamma\left(d^{*}\right) a_{d^{*}}
\end{aligned}
$$

with

$$
\Theta\left(\ell_{1}, \ell_{2}\right)=\int_{0 \leq t_{1}, t_{2} \leq 1} w^{\prime}\left(t_{1}\right) w^{\prime}\left(t_{2}\right) \log ^{+\kappa} \min \left(Q^{t_{1}} / \ell_{1}, Q^{t_{2}} / \ell_{2}\right) d t_{1} d t_{2}
$$

and where $\log ^{+\kappa} x=(\log \max (1, x))^{\kappa}$.

\section{The influence of $\mathcal{K}^{*}$}

The influence of the compact set $\mathcal{K}^{*}$ in (6.1) is contained in the function $\gamma$. In our main application, we will have $\gamma(p)=1 / p$, but we can encompass a more general case without much more difficulty. We assume there exists $\kappa^{\prime}>0$ such that

$$
\sum_{d^{*} /\left(d^{*}, \mathfrak{f}_{0}\right)=1}\left|\gamma\left(d^{*}\right)-\frac{\kappa^{\omega\left(d^{*}\right)}}{d^{*}}\right| \tau\left(d^{*}\right)^{2}\left|a_{d^{*}}\right|=\mathcal{O}(1 / R) .
$$

We could put $o(1)$ (i.e. a function that goes to 0 when $P_{0}$ and $P$ tend to infinity) instead of $\mathcal{O}(1 / R)$, but we would be blocked at section 13 . Hypothesis $\left(H_{6}\right)$ implies that $S_{0}^{(2)}\left(\left(a_{d^{*}}\right)_{d^{*} \leq D^{*}}\right)$ equals $S_{0}^{(3)}\left(\left(a_{d^{*}}\right)_{d^{*} \leq D^{*}}\right)$ up to an error term of size $\mathcal{O}\left(\left\|w^{\prime}\right\|_{\infty}^{2} /(R Z)\right)$, where

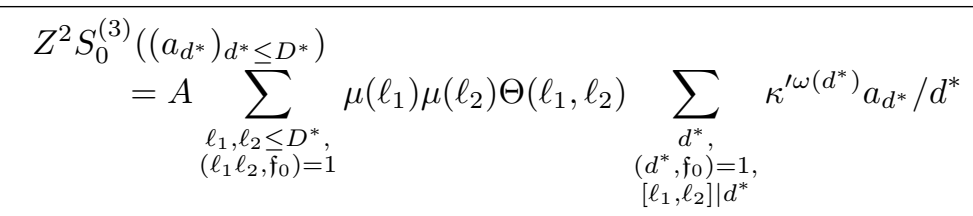




\section{Some lemmas on classical sums over primes}

To save on typography, in this section, a sum with a $*$ superscript is restricted to all its prime variables to be $>P_{0}$. We define classically

$$
\operatorname{li}(x)=\int_{2}^{x} \frac{d t}{\log t}
$$

This function is sometimes called the Eulerian logarithmic integral and should not be confused with the logarithmic integral $\mathrm{Li}$, see $[\mathbf{1}, 5.1 .3]$, from which it differs by a constant. We prove some more results than necessary, but the general case is not much more difficult than the special one we shall require, and these estimates prepare the ground for not only using $a_{r}[P]$ but the sequence of square-free integers $\leq P$ that have exactly $r$ prime factors, each of them being more than $P_{0}$.

\subsection{A general result}

In dimension 1 , we set

$$
W(\phi, t)=\max _{P_{0} \leq y \leq t}\left(|\phi(y)|+\frac{\log y}{y} \int_{P_{0}}^{y} \frac{x\left|\phi^{\prime}(x)\right| d x}{\log x}\right)
$$

while, in dimension $\nu$, we set $W_{\nu}(\phi, t)$ to be

$$
\max _{\substack{P_{0} \leq y_{1}, \cdots, y_{\nu} \leq t \\ y_{1} y_{2} \cdots y_{\nu} \leq t}}\left(\left|\phi\left(y_{1}, \cdots, y_{\nu}\right)\right|+\sum_{1 \leq j \leq \nu} \frac{\log y_{j}}{y_{j}} \int_{P_{0}}^{y_{j}} \frac{x\left|D_{j}\left(\phi, y_{1}, \cdots, y_{\nu} ; x\right)\right| d x}{\log x}\right)
$$

where $D_{j}\left(\phi, y_{1}, \cdots, y_{\nu} ; x\right)$ denotes $\partial \phi / \partial y_{j}\left(y_{1}, \cdots, y_{j-1}, x, y_{j+1}, \cdots, y_{\nu}\right)$.

Lemma 8.1. Let $\phi$ be a continuous function $C^{1}$ per pieces. For every $B \geq 1$, we have

$$
\sum_{p \leq t}^{*} \phi(p)=\int_{P_{0}}^{t} \frac{\phi(t) d t}{\log t}+\mathcal{O}_{B}\left(\frac{t W(\phi, t)}{R^{B} \log t}\right) .
$$

Proof. Let us denote by $E$ the (inside of the) error term above.

$$
\begin{aligned}
\sum_{p \leq t}^{*} \phi(p) & =\sum_{p \leq t}^{*}\left(-\int_{p}^{t} \phi^{\prime}(x) d x+\phi(t)\right) \\
& =\left(\operatorname{li}(t)-\operatorname{li}\left(P_{0}\right)\right) \phi(t)-\int_{P_{0}}^{t}\left(\operatorname{li}(x)-\operatorname{li}\left(P_{0}\right)\right) \phi^{\prime}(x) d x+\mathcal{O}(E) \\
& =\int_{P_{0}}^{t} \phi(t) \frac{d t}{\log t}+\mathcal{O}(E) .
\end{aligned}
$$

Lemma 8.2. Let $\phi\left(x_{1}, x_{2}, \cdots, x_{\nu}\right)$ be a continuous function over $\mathbb{R}^{+\nu}$ which is $C^{1}$ by piece. We have, for every $B \geq 1$,

$$
\begin{aligned}
\sum_{p_{1} \cdots p_{\nu} \leq t}^{*} \phi\left(p_{1}, \cdots, p_{\nu}\right)=\int_{\substack{P_{0} \leq x_{1}, \cdots, x_{\nu} \leq t, x_{1} \cdots x_{\nu} \leq t}} \frac{\phi\left(x_{1}, \cdots, x_{\nu}\right) d x_{1} \cdots d x_{\nu}}{\log x_{1} \cdots \log x_{\nu}} \\
+\mathcal{O}_{B}\left(\frac{t(\log \log t)^{\nu-1}}{R^{B} \log t} W_{\nu}(\phi, t)\right) .
\end{aligned}
$$


Proof. We proceed by induction.

Here is the induction step. We write $t=T^{\prime} T^{\prime \prime}$ and it is enough to select $T^{\prime}=T^{\prime \prime}=\sqrt{t}$. Let $S_{\nu+1}(\phi, t)$ be the sum to evaluate. The Dirichlet hyperbola principle gives us

$$
\begin{aligned}
S_{\nu+1}(\phi, t)= & \sum_{p_{1} p_{2} \cdots p_{\nu} \leq T^{\prime}}^{*} S_{1}\left(\phi\left(p_{1}, \cdots, p_{\nu}, \cdot\right), t /\left(p_{1} p_{2} \cdots p_{\nu}\right)\right) \\
& +\sum_{p_{\nu+1} \leq T^{\prime \prime}}^{*}\left(S_{\nu}\left(\phi\left(\cdot, \cdots, \cdot p_{\nu+1}\right), t / p_{\nu+1}\right)-S_{\nu}\left(\phi\left(\cdot, \cdots, \cdot, p_{\nu+1}\right), T^{\prime}\right)\right) .
\end{aligned}
$$

Concerning the first sum, say $\Sigma_{1}$, we use Lemma 8.1 to get

$$
\begin{aligned}
\Sigma_{1}=\int_{P_{0}}^{t} S_{\nu}\left(\phi\left(\cdot, \cdots, \cdot, x_{\nu+1}, \min \left(T^{\prime}, t / x_{\nu+1}\right)\right)\right. & \frac{d x_{\nu+1}}{\log x_{\nu+1}} \\
& +\mathcal{O}_{B}\left(\sum_{p_{1} \cdots p_{\nu} \leq T^{\prime}}^{*} \frac{t}{R^{B} p_{1} \cdots p_{\nu} \log T^{\prime \prime}} W_{\nu+1}(\phi, t)\right) .
\end{aligned}
$$

The error term is admissible. We use the induction hypothesis on the main term, say $\Sigma_{1,1}$, and get

$$
\begin{array}{r}
\Sigma_{1,1}=\int_{P_{0}}^{t} \int_{\begin{array}{r}
P_{0} \leq x_{1}, \cdots, x_{\nu} \leq \min \left(T^{\prime}, t / x_{\nu+1}\right) \\
x_{1} \cdots x_{\nu} x_{\nu+1} \leq t
\end{array}} \phi\left(x_{1}, \cdots, x_{\nu}, x_{\nu+1}\right) \prod_{1 \leq i \leq \nu+1} \frac{d x_{i}}{\log x_{i}} \\
\quad+\mathcal{O}_{B}\left(\int_{P_{0}}^{t} \frac{t}{x_{\nu+1} \log T^{\prime} \log x_{\nu+1}} \frac{(\log \log t)^{\nu-2}}{R^{B}} W_{\nu+1}(\phi, t) d t\right) .
\end{array}
$$

The error term is again admissible. We handle the other part in pretty much the same manner and get the claimed lemma.

Lemma 8.3. Let $\phi\left(x_{1}, x_{2}, \cdots, x_{\nu}\right)$ be a continuous function over $\mathbb{R}^{+\nu}$ which is $C^{1}$ by piece. We have, for every $B \geq 1$,

$$
\begin{gathered}
\sum_{p_{1} \cdots p_{\nu} \leq t}^{*} \frac{\phi\left(p_{1}, p_{2}, \cdots, p_{\nu}\right)}{p_{1} \cdots p_{\nu}}=\int_{\begin{array}{c}
P_{0} \leq x_{1}, x_{2}, \cdots, x_{\nu} \leq t \\
x_{1} x_{2} \cdots x_{\nu} \leq t
\end{array}} \frac{\phi\left(x_{1}, x_{2}, \cdots, x_{\nu}\right) d x_{1} \cdots d x_{\nu}}{x_{1} \cdots x_{\nu} \log x_{1} \cdots \log x_{\nu}} \\
+\mathcal{O}_{B}\left(\frac{(\log \log t)^{\nu-1}}{R^{B}} W_{\nu}(\phi, t)\right) .
\end{gathered}
$$

\subsection{Application}

Theorem 8.1. We have, with $R=\log P_{0}$ and for every $B \geq 1$,

$$
\sum_{\substack{p_{1} p_{2} \cdots p_{\nu} \leq t, P_{0}<p_{1}, \cdots, p_{\nu}}} 1=\frac{t}{\log t} A_{\nu}\left(\frac{\log t}{R}\right)+\mathcal{O}_{B}\left(\frac{t(\log \log t)^{\nu-1}}{R^{B} \log t}\right)
$$

where $A_{\nu}$ is a $C^{\infty}\left[\nu, \infty\left[\right.\right.$-function such that $A_{\nu}(u)=0$ when $u \leq \nu$ and

$$
0 \leq A_{\nu}(u) \ll_{\nu}(\log u)^{\nu-1}, \quad 0 \leq A_{\nu}^{\prime}(u) \ll_{\nu}(\log u)^{\nu-1} / u
$$

It is defined recursively by

$$
A_{\nu+1}(u)=\int_{\nu}^{u-1} A_{\nu}(v) \frac{u d v}{v(u-v)}=\int_{\nu}^{u-1}\left(\frac{A_{\nu}(v)}{v}+\frac{A_{\nu}(v)}{u-v}\right) d v .
$$

We have, when $u \geq 1$,

$$
A_{1}(u)=1
$$


and when $u \geq 2$,

$$
A_{2}(u)=2 \log (u-1) .
$$

THEOREM 8.2. We have, when $\nu \in\{1,2,3\}$ and for every $B \geq 1$ :

$$
\sum_{\substack{p_{1} \cdots p_{\nu} \leq t, p_{1}, \cdots, p_{\nu}>P_{0}}} \frac{1}{p_{1} \cdots p_{\nu}}=\nu ! \mathscr{F}_{\nu}\left(\frac{\log t}{\log P_{0}}\right)+\mathcal{O}_{B}\left(\frac{(\log \log t)^{\nu}}{R^{B}}\right)
$$

where $\mathscr{F}_{\nu}$ is given when $h \geq \nu$ by:

$$
\left\{\begin{array}{l}
\mathscr{F}_{1}(h)=\log h \\
\mathscr{F}_{2}(h)=\int_{2}^{h} \log (v-1) \frac{d v}{v} \\
\mathscr{F}_{3}(h)=\frac{1}{3} \int_{2}^{h-1} \log (v-1) \log (h-v) \frac{d v}{v} .
\end{array}\right.
$$

We further set $\mathscr{F}_{0}(h)=1$.

A direct approach (without using Theorem 8.1) seems to give one less $\log \log t$ in the error term. This is irrelevant for our applications.

\section{Specialisation of $a_{d^{*}}$ and summing over $d^{*}$}

The expression (7.1) for our main term still contains three variables, $\ell_{1}, \ell_{2}$ and $d^{*}$, and we discuss here our treatment of the variable $d^{*}$. We have to restrict $a_{d^{*}}$ to $a_{r}[P]$ to do so. To simplify notations, we tag our summation signs with a $\star$ to indicate that all the prime factors of the variables therein are within $\left(P_{0}, P\right]$. We add the simplistic hypothesis

$$
\left\{\begin{array}{l}
\gamma(p) \ll 1 / p \\
\sum_{p \geq P_{0}}\left|\gamma(p)-\frac{\kappa^{\prime}}{p}\right|=\mathcal{O}\left(1 / \log P_{0}\right)
\end{array}\right.
$$

Hypotheses $\left(H_{5}\right)$ and $\left(H_{6}\right)$ are then trivially verified. Since $\frac{\log P}{\log P_{0}}=\tau / \varepsilon$, we find that

$$
\sum_{\substack{d^{*},\left(d^{*}, \mathfrak{f}_{0}\right)=1, m \mid d^{*}}} \kappa^{\prime \omega\left(d^{*}\right)} a_{d^{*}} / d^{*}=\frac{\kappa^{\prime r}}{m} \frac{\log (\tau / \varepsilon)^{r-\omega(m)}}{(r-\omega(m)) !}+\mathcal{O}\left(\log (\tau / \varepsilon)^{r-\omega(m)} /\left(m P_{0}\right)\right)
$$

(this is valid even if $\omega(m)=r$ ) where $H=(\log P) / R$. As a consequence, and replacing $\ell_{1}$ of $\ell_{0} \ell_{1}$ and $\ell_{2}$ by $\ell_{0} \ell_{2}$ with $\operatorname{gcd}\left(\ell_{0} \ell_{1}, \ell_{0} \ell_{2}\right)=\ell_{0}$, we find that

$$
\begin{aligned}
& Z^{2} S_{0}^{(3)}\left(a_{r}[P]\right)= \\
& A \kappa^{\prime r} \sum_{\substack{\ell_{0}, \ell_{1}, \ell_{2}, \omega\left(\ell_{0} \ell_{1} \ell_{2}\right) \leq r}}^{\star} \frac{\mu\left(\ell_{1}\right) \mu\left(\ell_{2}\right) \mu^{2}\left(\ell_{0} \ell_{1} \ell_{2}\right)}{\ell_{0} \ell_{1} \ell_{2}} \Theta\left(\ell_{0} \ell_{1}, \ell_{0} \ell_{2}\right) \frac{\log (\tau / \varepsilon)^{r-\omega\left(\ell_{0} \ell_{1} \ell_{2}\right)}}{\left(r-\omega\left(\ell_{0} \ell_{1} \ell_{2}\right)\right) !} \\
& +\mathcal{O}\left(Z \log (\tau / \varepsilon)^{2 r} / P_{0}\right)
\end{aligned}
$$

10. Summing over $\ell_{0}, \ell_{1}$ and $\ell_{2}$

We only have the last three variables $\ell_{0}, \ell_{1}$ and $\ell_{2}$ to take care of. The fact that the weight $w$ is kept general complicates things somewhat but in fact selecting $w(t)=\max (0,1-t)$ would 
not lead to notoriously simpler expression. Let us define

$$
\mathscr{G}_{s}^{(0)}(P)=\sum_{\substack{\ell_{0}, \ell_{1}, \ell_{2}, \omega\left(\ell_{0} \ell_{1} \ell_{2}\right)=s}}^{\star} \frac{\mu\left(\ell_{1}\right) \mu\left(\ell_{2}\right) \mu^{2}\left(\ell_{0} \ell_{1} \ell_{2}\right)}{\ell_{0} \ell_{1} \ell_{2}} \Theta\left(\ell_{0} \ell_{1}, \ell_{0} \ell_{2}\right)
$$

On recalling $(6.2)$, we find that

$$
\mathscr{G}_{s}^{(0)}(P)=(\log Q)^{\kappa} \int_{0 \leq t_{1}, t_{2} \leq 1} w^{\prime}\left(t_{1}\right) w^{\prime}\left(t_{2}\right) \mathscr{G}_{s}^{(1)}\left(t_{1}, t_{2}, P\right) d t_{1} d t_{2}
$$

with

$$
\begin{gathered}
\mathscr{G}_{s}^{(1)}\left(t_{1}, t_{2}, P\right)= \\
\sum_{\substack{\ell_{0} \ell_{1} \leq Q^{t_{1}} \\
\ell_{0} \ell_{2} \leq Q^{t_{2}}, \omega\left(\ell_{0} \ell_{1} \ell_{2}\right)=s}}^{\star} \frac{\mu\left(\ell_{1}\right) \mu\left(\ell_{2}\right) \mu^{2}\left(\ell_{0} \ell_{1} \ell_{2}\right)}{\ell_{0} \ell_{1} \ell_{2}} \min \left(t_{1}-\alpha \log \left(\ell_{0} \ell_{1}\right), t_{2}-\alpha \log \left(\ell_{0} \ell_{2}\right)\right)^{\kappa} .
\end{gathered}
$$

Lemma 8.3 is tailored to handle this sum. We thus find that

$$
\begin{gathered}
\mathscr{G}_{s}^{(1)}\left(t_{1}, t_{2}, P\right)= \\
\sum_{b+c+d=s} \frac{(-1)^{c+d}}{b ! c ! d !} \int_{P_{0} \leq x_{1}, \cdots, x_{b} \leq P} \int_{P_{0} \leq y_{1}, \cdots, y_{c} \leq P} \int_{P_{0} \leq z_{1}, \cdots, z_{d} \leq P} \\
\frac{\min \left(t_{1}-\alpha \log \left(\prod_{i} x_{i} \prod_{i} y_{i}\right), t_{2}-\alpha \log \left(\prod_{i} x_{i} \prod_{i} z_{i}\right)\right)^{+\kappa} \prod_{i} d x_{i} \prod_{i} d y_{i} \prod_{i} d z_{i}}{\prod_{i}\left(x_{i} \log x_{i}\right) \prod_{i}\left(y_{i} \log y_{i}\right) \prod_{i}\left(z_{i} \log z_{i}\right)} \\
+\mathcal{O}_{B}\left(\frac{(\log \log P)^{s-1}}{R^{B}}\right) .
\end{gathered}
$$

This calls for a several changes of variables, which when done, lead to

$$
\mathscr{G}_{s}^{(1)}\left(t_{1}, t_{2}, P\right)=\sum_{b+c+d=s} \frac{(-1)^{c+d}}{b ! c ! d !} \mathscr{G}_{b, c, d}^{(2)}\left(t_{1}, t_{2}, \varepsilon, \tau\right)+\mathcal{O}_{B}\left(\frac{(\log \log P)^{s-1}}{R^{B}}\right)
$$

where

$$
\mathscr{G}_{b, c, d}^{(2)}\left(t_{1}, t_{2}, \varepsilon, \tau\right)=\int_{\begin{array}{c}
\varepsilon \leq u_{1}, \cdots, u_{b} \leq \tau \\
\left(\min \left(t_{1}-\Sigma_{i} v_{i}, t_{2}-\Sigma_{i} w_{i}\right)-\Sigma_{i} u_{i}\right)^{+\kappa} \prod_{i} d u_{i} \prod_{i} d v_{i} \prod_{i} d w_{i}
\end{array}} \int_{\prod_{i} u_{i} \prod_{i} v_{i} \prod_{i} w_{i}}
$$

We thus find that $\left.S_{0}^{(3)}\left(a_{r}[P]\right)\right)$ equals $S_{0}^{(4)}\left(a_{r}[P]\right)$ up to an error term of size $\mathcal{O}_{B}\left((\log \log P)^{r} /\left(R^{B} Z\right)\right)$, where

$$
\begin{aligned}
& Z S_{0}^{(4)}\left(a_{r}[P]\right) \\
& \quad=A \kappa^{\prime r} \int_{0 \leq t_{1}, t_{2} \leq 1} w^{\prime}\left(t_{1}\right) w^{\prime}\left(t_{2}\right) \mathfrak{G}_{\kappa, r}\left(t_{1}, t_{2}, \varepsilon, \tau\right) d t_{1} d t_{2}
\end{aligned}
$$

and

$$
\mathfrak{G}_{\kappa, r}\left(t_{1}, t_{2}, \varepsilon, \tau\right)=\sum_{a+b+c+d=r} \frac{(-1)^{c+d}}{a ! b ! c ! d !} \mathscr{G}_{b, c, d}^{(2)}\left(t_{1}, t_{2}, \varepsilon, \tau\right) \log (\tau / \varepsilon)^{a} .
$$

This quantity depends on $P_{0}$, while the quantity we computed is independent from it. This problem is cleared in the subsequent sections. 


\section{Average decay rate of the Fourier transforms of piecewise affine linear functions on $\mathbb{R}^{n}$}

This part is due to D.S. Ramana [18] whom we thank warmly for his input as well as for the authorization to reproduce his argument below.

A $k$-simplex in $\mathbb{R}^{n}$ is the convex hull of $k+1$ affine linearly independent points in $\mathbb{R}^{n}$, where $k$ and $n$ are integers. If a simplex $P$ is spanned by a set $S$ then the simplices spanned by the proper subsets of $S$ are called the faces of $P$.

The union of the faces of a simplex is called the boundary of the simplex. The complement in a simplex of its boundary is called its interior. When the simplex is an $n$-simplex in $\mathbb{R}^{n}$, its boundary and interior are same its boundary and interior as a subset of the topological space $\mathbb{R}^{n}$.

By a simplicial complex in $\mathbb{R}^{n}$ we shall mean a finite family $\mathcal{K}$ of simplexes such that every face of a simplex in $\mathcal{K}$ is also in $\mathcal{K}$ and the intersection of any two simplexes $A$ and $B$ in $\mathcal{K}$ is a face of both $A$ and $B$. This implies, in particular, that interiors of distinct simplices in $\mathcal{K}$ are disjoint. The union of the simplexes in a simplicial complex $\mathcal{K}$ is denoted by $|\mathcal{K}|$. A compactly supported complex valued function on $\mathbb{R}^{n}$ is said to piecewise affine linear if its support is contained in $|\mathcal{K}|$, for some simplicial complex $\mathcal{K}$, and if its restriction to any simplex in $\mathcal{K}$ is affine linear.

THEOREM 11.1. If $n$ is an integer $\geq 1$ and $f$ is a continuous compactly supported complex valued function on $\mathbb{R}^{n}$ that is piecewise affine linear on a simplicial complex $\mathcal{K}$ we then have that, the Fourier transform $\hat{f}$ of $f$ satisfies

$$
\int_{\mathbb{R}^{n}} \frac{\|x\|^{n}|\hat{f}(x)|}{\log (2+\|x\|)^{n+1}} d x \ll M m,
$$

where $M$ is the essential supremum of $\|\nabla f\|$ on $|\mathcal{K}|, m$ is the number of $n$-simplexes in $\mathcal{K}$ and implicit constant depends only on $n$.

The Fejér kernel in dimension 1 shows that the power $n$ is optimal.

We will infer this Theorem from (ii) of Theorem 2.1 of [3], which gives the average rate of decay of the Fourier transform of the characteristic function of a polyhedron, by an application of the divergence theorem.

Preliminaries Let us verify that in any simplicial complex $\mathcal{K}$ every $(n-1)$-simplex of $\mathcal{K}$ is either the common face of exactly two $n$-simplexes of $\mathcal{K}$ or lies on the boundary of $|\mathcal{K}|$.

For any $(n-1)$-simplex $F$ in $\mathbb{R}^{n}$ there is a unique affine linear form $H$ such that $F$ lies on the (affine) hyperplane defined by $H(x)=0$. By abuse of notation, we call the half-spaces defined by the inequalities $H(x) \geq 0$ and $H(x) \leq 0$ the sides of $F$. Now suppose that $P$ and $P^{\prime}$ are $n$-simplexes in $\mathbb{R}^{n}$ whose intersection is an $(n-1)$-simplex $F$ that is a face of both $P$ and $P^{\prime}$. If both these simplexes lie on the same side of $F$, their interiors have a non-empty intersection. Indeed, there are $n+1$ affine linear forms $H_{i}$ and $H_{i}^{\prime}$ such that $P$, respectively $P^{\prime}$, is the set of $x$ in $\mathbb{R}^{n}$ that satisfy $H_{i}(x) \geq 0$, resp. $H_{i}^{\prime}(x) \geq 0$, for $1 \leq i \leq n+1$. Suppose that $H_{1}=H_{1}^{\prime}$ and that the face $F$ lies on the hyperplane determined by these linear forms. Let $p$ be a point in the interior of $F$. Then $F$ is the unique face of either $P$ or $P^{\prime}$ that contains $p$. Thus $x=p$ satisfies the inequalities $H_{i}(x)>0$ and $H_{i}^{\prime}(x)>0$ for all $i \geq 2$. These strict inequalities define an open set $U$ in $\mathbb{R}^{n}$ that contains $p$. Since $p$ lies on the boundary of the open half-space determined by $H_{1}(x)>0, U$ has a non-empty intersection $V$ with this half-space. Plainly, $V$ is contained in the interior of both $P$ and $P^{\prime}$. It follows that if $P$ and $P^{\prime}$ are $n$-simplexes of a simplicial complex and if they have a common face $F$ that is an $(n-1)$-simplex, then, since their interiors do not intersect, $P$ and $P^{\prime}$ must necessarily lie on distinct sides of the face $F$. This implies that 
an $(n-1)$-simplex $F$ can be the common face of no more than two $n$-simplexes of a simplicial complex.

Let us now suppose that $F$ is an $(n-1)$-simplex that is the face of a unique $n$-simplex $P$ of a simplicial complex $\mathcal{K}$. Let $p$ be a point in the interior of $F$. Suppose that $p$ lies in the interior of $|\mathcal{K}|$ and let $U$ be any open ball in $\mathbb{R}^{n}$ centered at $p$ and contained in $|\mathcal{K}|$. Since $p$ lies on the boundary of $P$, the intersection $V$ of $U$ with the complement of $P$ in $\mathbb{R}^{n}$ is a nonempty open subset of $\mathbb{R}^{n}$. Since $V$ is contained in the complement of $P$ in $|\mathcal{K}|$, it follows that this complement has a non-empty interior and therefore contains an $n$-simplex whose interior intersects $U$. Since the number of $n$-simplexes in $\mathcal{K}$ is finite, we see on taking for $U$ any open ball in a decreasing sequence of such balls centered at $p$ and contained in $|\mathcal{K}|$ that there is an $n$-simplex $P^{\prime}$ contained in $\mathcal{K}$, distinct from $P$, and containing $p$. Since $p$ is in the interior of $F$ this means that $F$ is a face of $P^{\prime}$, contradicting our hypothesis on $F$. Thus every point in interior of $F$ lies on the boundary of $|\mathcal{K}|$ and consequently $F$ lies on the boundary of $|\mathcal{K}|$ as well, as required.

Finally, we recall the divergence theorem. If $v$ is a $C^{1}$ function from an open set $\Omega$ in $\mathbb{R}^{n}$ to $\mathbb{R}$, its gradient is the function from $\Omega$ into $\mathbb{R}^{n}$ defined by $t \mapsto\left(\frac{\partial v}{\partial t_{1}}, \ldots, \frac{\partial v}{\partial t_{n}}\right)$ and is denoted by $\nabla v$. If $v$ is a $C^{1}$ function from an open set $\Omega$ into $\mathbb{R}^{n}$, its divergence is the function from $\Omega$ into $\mathbb{R}$ defined by $t \mapsto \frac{\partial v}{\partial t_{1}}+\ldots+\frac{\partial v}{\partial t_{n}}$ and is denoted by $\nabla \cdot v$. Please note the presence of the "." symbol.

Let $F$ be an $(n-1)$-simplex $F$ that is the face of an $n$-simplex $P$ in $\mathbb{R}^{n}$. Suppose that $F$ lies on a hyperplane $H$ in $\mathbb{R}^{n}$ determined by the relation $\langle t, c\rangle+a=0$, for some $c$ of norm 1 in $\mathbb{R}^{n}$ and some $a$ in $\mathbb{R}^{n}$. If $P$ lies on the side given by $\langle t, c\rangle+a \leq 0$ then the unit outward normal $n_{F}$ to $F$ is defined to be $c$. When $P$ lies on the other side of $F$ we set $n_{F}=-c$.

Suppose that $P$ is an $n$-simplex in $\mathbb{R}^{n}$ and $v\left(t_{1}, \ldots, t_{n}\right)$ is a $C^{1}$ function from an open neighbourhood of $P$ into $\mathbb{R}^{n}$. Then the divergence theorem is the relation

$$
\int_{P} \nabla \cdot v d t_{1} d t_{2} \ldots d t_{n}=\sum_{F \in \mathcal{F}(P)} \int_{F}\left\langle v, n_{F}\right\rangle d S_{F}
$$

where $\mathcal{F}(P)$ denotes the set of $(n-1)$ simplexes that are the faces of $P, n_{F}$ denotes the unit outward normal and $d S_{F}$ denotes the element of $(n-1)$ dimensional surface measure for each such face $F$. To describe $d S_{F}$ in terms of a parametrisation (co-ordinate chart) of a given face $F \in \mathcal{F}(P)$ of a simplex $P$, let $\phi$ from $\mathbb{R}^{n-1}$ to the hyperplane containing $F$ be a parametrisation. Then from [22, page 86] we have

$$
d S_{F}=\sqrt{\operatorname{det}\left((D \phi)^{t}(D \phi)\right)} d t_{1} d t_{2} \ldots d t_{n-1} .
$$

If $g$ is an orthogonal matrix that transports $n_{F}$ to the vector $(0,0, \ldots, 0,1)$ and let $a$ in $\mathbb{R}^{n}$ be such that $t \mapsto g t+a$ is an affine linear isomorphism from the co-ordinate plane $t_{n}=0$ to the hyperplane containing $F$, the composing this map with the embedding $t \mapsto(t, 0)$ from $\mathbb{R}^{n-1}$ onto the co-ordinate hyperplane $t_{n}=0$, we get a parametrisation $\phi$. With this parametrisation we have

$$
d S_{F}=d t_{1} d t_{2} \ldots d t_{n-1}
$$

since $D \phi$ is given by the matrix of $n-1$ columns and $n$ rows whose columns are the first $n-1$ columns of $g$, which is orthogonal. Let us note that since $\phi$ is affine linear, $\phi^{-1}(F)$ is a simplex in $\mathbb{R}^{n-1}$.

Proof of Theorem 11.1 We begin by noting that if $\phi(t)$ and $f(t)$, with $t=\left(t_{1}, t_{2}, \ldots, t_{n}\right)$ are $C^{1}$ functions on an open set in $\mathbb{R}^{n}$ then at any point $t$ in this open set with $\nabla(\phi(t)) \neq 0$ 
we have

$$
\nabla \cdot\left(\frac{\nabla \phi}{\|\nabla \phi\|^{2}} f(t) e(\phi(t))\right)=2 \pi i f(t) e(\phi(t))+\nabla \cdot\left(\frac{\nabla \phi}{\|\nabla \phi\|^{2}} f(t)\right) e(\phi(t)) .
$$

We apply the above relation with $\phi(t)=\langle x, t\rangle$, where $x \neq 0$ is a given point in $\mathbb{R}^{n}$. Then $\nabla \phi$ is $x$ and we have from (11.5) that

$$
2 \pi i f(t) e(\langle x, t\rangle)=\nabla \cdot\left(\frac{x}{\|x\|^{2}} f(t) e(\langle x, t\rangle)\right)-\frac{\langle x, \nabla f\rangle}{\|x\|^{2}} e(\langle x, t\rangle) .
$$

Let $P$ denote any $n$-simplex in $\mathcal{K}$. We then integrate the above relation over $P$ and sum over all $n$-simplexes $P$ in $\mathcal{K}$. On now recalling that the support of $f$ is in $|\mathcal{K}|$, that the interiors of distinct simplexes in $\mathcal{K}$ are disjoint and that the complement in $\mathcal{K}$ of the union of the $n$-simplexes in $\mathcal{K}$ is of measure zero, we conclude that

$$
2 \pi i \hat{f}(x)=\sum_{P \in \mathcal{K}} \int_{P} \nabla \cdot\left(\frac{x f(t)}{\|x\|^{2}} e(\langle x, t\rangle)\right) d t-\sum_{P \in \mathcal{K}} \int_{P} \frac{\langle x, \nabla f\rangle}{\|x\|^{2}} e(\langle x, t\rangle) d t .
$$

The divergence theorem in the form given, for example, by [22, Théorème 6.10.10, page 365], applied to each $P$ in $\mathcal{K}$ gives the following expression for the left hand side of (11.7).

$$
\sum_{P \in \mathcal{K}} \int_{P} \nabla \cdot\left(\frac{x f(t)}{\|x\|^{2}} e(\langle x, t\rangle)\right) d t=\sum_{P \in \mathcal{K}} \sum_{F \in \mathcal{F}(P)} \int_{F} \frac{\left\langle x, n_{F}\right\rangle}{\|x\|^{2}} f(t) e(\langle x, t\rangle) d S_{F} .
$$

where, for each $n$-simplex in $\mathcal{K}$, we have written $\mathcal{F}(P)$ to denote the set of $(n-1)$-simplexes in $\mathcal{K}$ that are faces of $P$ and used $n_{F}$ for the unit outward normal, $d S_{F}$ for the surface measure of a given face $F$ in $\mathcal{F}(P)$.

Let $F$ be an $(n-1)$-simplex in $\mathcal{K}$. If $F$ is a common face of $n$-simplexes $P$ and $P^{\prime}$ and $n_{F}$ is the unit outward normal to $F$ as a face of $P$ then the unit outward normal to $F$ as a face of $P^{\prime}$ is $-n_{F}$. We have already seen that $F$ can be a common face of exactly two $n$-simplexes in $\mathcal{K}$. Since $f$ is a continuous function, it follows that the sum of the contributions to the right hand side of (11.8) from $F$ that are common faces of pairs of $n$-simplexes is zero. If $F$ is not the common face of two $n$-simplexes, it must necessarily lie on the boundary of $|\mathcal{K}|$, where $f$ vanishes, since it is continuous and its support is in $|\mathcal{K}|$. Thus the left hand side of (11.7) is zero. This proof is in fact the adaptation in several variables of the usual one concerning the Fejér kernel $(1-|t|)^{+}$: to show that its Fourier transform is small enough, we use integration by parts and show the integrated term vanishes because of continuity.

Turning to the second term on the right hand side of (11.7), we note that since $f$ is piecewise linear on $\mathcal{K}, \nabla f$ is a constant on each $P$. Moreover, the Cauchy-Schwarz inequality gives $|\langle x, \nabla f\rangle| \leq\|x\|\|\nabla f\| \leq M\|x\|$. It now follows from (11.7) and the triangle inequality that

$$
2 \pi|\hat{f}(x)| \leq \frac{M}{\|x\|} \sum_{P \in \mathcal{K}}\left|\int_{P} e(\langle x, t\rangle) d t\right| .
$$

This equation yields a good bound for $\hat{f}(x)$ when $x$ is not orthogonal to any $P$, or close to be so, but fails in that case. We need an $L^{1}$ estimate, and in that case, the measure of the set of "bad cases" is small enough. We proceed to give a rigorous content to this remark. Integrating (11.9) over $\mathbb{R}^{n}$ and passing to polar co-ordinates, i.e., recalling that $\mathbb{R}^{n}=\mathbb{R}_{+} \times S^{n-1}(\mathbb{R})$ and applying Fubini's theorem, we deduce that

$$
\begin{aligned}
& \int_{\mathbb{R}^{n}} \frac{\|x\|^{n}|\hat{f}(x)|}{\log (2+\|x\|)^{n+1}} d x \\
& \ll M \sum_{P \in \mathcal{K}} \int_{0}^{\infty} \frac{\lambda^{n-1}}{\log (2+\lambda)^{n+1}} \int_{S^{n-1}(\mathbb{R})}\left|\int_{P} e(\langle\lambda \sigma, t\rangle) d t\right| d \sigma d \lambda
\end{aligned}
$$


where $d \sigma$ is the surface measure on $S^{n-1}(\mathbb{R})$. From (ii), Theorem 2.1 of [3], as also from [25, pages 124-125], we have for any simplex $P$ in $\mathcal{K}$ that

$$
\int_{S^{n-1}(\mathbb{R})}\left|\int_{P} e(\langle\lambda \sigma, t\rangle) d t\right| d \sigma \ll \frac{\log (1+\lambda)^{n-1}}{(1+\lambda)^{n}} .
$$

On substituting this bound into (11.10) and integrating over $\lambda$ we conclude the proof of the theorem except for one detail - it is necessary to check that the method of proof in [3] gives us (11.11) with an implied constant that depends only on $n$. This is easily seen by following the argument on pages 257-259 of [3], which is an induction on $n$ through an application of the divergence theorem to the integral over $P$ in (11.11), and remarking that, for any $n \geq 1$, the left hand side of (11.11) is invariant when $P$ is transformed by affine isometries of $\mathbb{R}^{n}$.

\section{Using a Fourier transform}

We define

$$
f_{t_{1}, t_{2}, \kappa}(u, v, w)=\max \left(0, \min \left(t_{1}-|u|-|v|, t_{2}-|u|-|w|\right)\right)^{\kappa},
$$

and $f_{t_{1}, t_{2}}=f_{t_{1}, t_{2}, 1}$. We expand $f_{t_{1}, t_{2}}$ as a Fourier transform, and we need to bound this Fourier transform uniformly in $t_{1}$ and $t_{2}$. It is possible to get such a proof on relying on the special form of $f$. Indeed Maxima [15] (which we have used via its graphical interface wxMaxima [27]) tells us that (with $X=2 \pi x, Y=2 \pi y, Z=2 \pi z$ and $t_{3}=t_{2}-t_{1}$ )

$$
\begin{gathered}
4 Y(Z+X-Y)(Z+X+Y) \cos \left(t_{3} Z+t_{1} X\right) \\
-4 Y(Z-X-Y)(Z-X+Y) \cos \left(t_{3} Z-t_{1} X\right) \\
+4 X(Z-X-Y)(Z+X-Y) \cos \left(t_{2} Z+t_{1} Y\right) \\
X Y Z \hat{f}_{t_{1}, t_{2}}(x, y, z)=\frac{-4 X(Z-X+Y)(Z+X+Y) \cos \left(t_{2} Z-t_{1} Y\right)}{(Z-X-Y)(Z+X+Y)(Z-X+Y)(Z+X-Y)}
\end{gathered}
$$

and

$$
\begin{gathered}
16 \cos \left(t_{3} Z\right) \cos \left(t_{1} X\right)-16 \cos \left(t_{2} Z\right) \cos \left(t_{1} Y\right) \\
-8\left(Z^{2}+Y^{2}-X^{2}\right) \sin \left(t_{3} Z\right) \sin \left(t_{1} X\right) /(Z X) \\
\hat{f}_{t_{1}, t_{2}}(x, y, z)=\frac{-8\left(Z^{2}-Y^{2}+X^{2}\right) \sin \left(t_{2} Z\right) \sin \left(t_{1} Y\right) /(Z Y)}{(Z-X-Y)(Z+X+Y)(Z-X+Y)(Z+X-Y)} .
\end{gathered}
$$

This last expression has the advantage of showing clearly what happens when $X, Y$ or $Z$ is close to 0 . Such a proof is however clumsy at best, rather lengthy and would not support any slight change in the initial function. In the course of this study, I formulated a conjecture that has been since proved by D.S. Ramana [18] and whose proof we reproduced in section 11 . Having this at hand, the general case is readily handled.

LEMMA 12.1. We have

$$
\int_{x, y, z}\left|\hat{f}_{t_{1}, t_{2}, \kappa}(x, y, z)\right|(1+|x|)^{\alpha}(1+|y|)^{\alpha}(1+|z|)^{\alpha} d x d y d z \ll 1
$$

provided $\alpha \in[0,1 / 3)$, and uniformly in $t_{1}$ and $t_{2}$ belonging to $[0,1]^{2}$. Note furthermore that $f_{t_{1}, 0, \kappa}=f_{0, t_{2}, \kappa}=0$.

Proof. We show that the convolution of any two functions that verify this inequality still verify this inequality. This will establish the required estimate by induction, the initial step being provided by Theorem 11.1 and the remark that we can bound $\left|\hat{f}_{t_{1}, t_{2}}(x, y, z)\right|$ by 1 when 
$x^{2}+y^{2}+z^{2} \leq 1$. This is however immediate by using the inequality

$$
(1+|h|)^{\alpha} \ll(1+|u|)^{\alpha}+(1+|h-u|)^{\alpha} .
$$

On expressing $f_{t_{1}, t_{2}, \kappa}$ in terms of its Fourier transform, we find that the function $\mathscr{G}_{b, c, d}^{(2)}\left(t_{1}, t_{2}, \varepsilon, \tau\right)$ is equal to

$$
\begin{aligned}
\int_{x, y, z} \hat{f}_{t_{1}, t_{2}, \kappa}(x, y, z) & \int_{\varepsilon \leq u_{1}, \cdots, u_{b} \leq \tau} \int_{\varepsilon \leq v_{1}, \cdots, v_{c} \leq \tau} \int_{\varepsilon \leq w_{1}, \cdots, w_{d} \leq \tau} \\
= & \prod_{i} \frac{e\left(x u_{i}\right) d u_{i}}{u_{i}} \prod_{i} \frac{e\left(y v_{i}\right) d v_{i}}{v_{i}} \prod_{i} \frac{e\left(z w_{i}\right) d w_{i}}{w_{i}} d x d y d z \\
= & \int_{x, y, z} \hat{f}_{t_{1}, t_{2}, \kappa}(x, y, z) E(\varepsilon, \tau, x)^{b} E(\varepsilon, \tau, y)^{c} E(\varepsilon, \tau, z)^{d} d x d y d z
\end{aligned}
$$

with

$$
E(\varepsilon, \tau, x)=\int_{\varepsilon \leq u \leq \tau} \frac{e(x u) d u}{u}=\log \frac{\tau}{\varepsilon}+E_{0}(\varepsilon, \tau, x),
$$

where

$$
E_{0}(\varepsilon, \tau, x)=\int_{\varepsilon \leq u \leq \tau} \frac{e(x u)-1}{u} d u .
$$

The change of variable $u \mapsto v=x u$ shows that $E_{0}(\varepsilon, \tau, x)$ is bounded in absolute value by $\mathcal{O}(\log (2+|x|))$. Replacing $E$ by $E_{0}$ yields the following formula:

$$
\begin{aligned}
\mathscr{G}_{b, c, d}^{(2)}\left(t_{1}, t_{2}, \varepsilon, \tau\right)= & \int_{x, y, z} \hat{f}_{t_{1}, t_{2}, \kappa}(x, y, z) \sum_{\ell, m, n \geq 0}\left(\begin{array}{l}
b \\
\ell
\end{array}\right)\left(\begin{array}{c}
c \\
m
\end{array}\right)\left(\begin{array}{l}
d \\
n
\end{array}\right) \\
& \left(\log \frac{\tau}{\varepsilon}\right)^{b+c+d-\ell-m-n} \mathscr{E}_{\ell, m, n}(\varepsilon, \tau, x, y, z) d x d y d z
\end{aligned}
$$

with the simplification:

$$
\mathscr{E}_{\ell, m, n}(\varepsilon, \tau, x, y, z)=E_{0}(\varepsilon, \tau, x)^{\ell} E_{0}(\varepsilon, \tau, y)^{m} E_{0}(\varepsilon, \tau, z)^{n} .
$$

This leads to (since $H=\tau / \varepsilon$ )

$$
\begin{aligned}
\mathfrak{G}_{\kappa, r}\left(t_{1}, t_{2}, \varepsilon, \tau\right)= & \int_{x, y, z} \hat{f}_{t_{1}, t_{2}, \kappa}(x, y, z) \sum_{a+b+c+d=r} \frac{(-1)^{b}}{a ! b ! c ! d !} \sum_{\ell, m, n \geq 0} \\
& \left(\begin{array}{l}
b \\
\ell
\end{array}\right)\left(\begin{array}{c}
c \\
m
\end{array}\right)\left(\begin{array}{c}
d \\
n
\end{array}\right)\left(\log \frac{\tau}{\varepsilon}\right)^{r-\ell-m-n} \mathscr{E}_{\ell, m, n}(\varepsilon, \tau, x, y, z) d x d y d z .
\end{aligned}
$$

It is almost immediate to replace $E_{0}(\varepsilon, \tau, \cdot)$ by $E_{0}(0, \tau, \cdot)$, by using Lemma 12.1 and the bound

$$
E_{0}(0, \tau, t)-E_{0}(\varepsilon, \tau, t) \ll \min (\varepsilon t, \log (2+|t|)) .
$$

We also need to note that, when $H \geq 1$,

$$
\int_{\substack{x, y, z \\|x| \geq H}}\left|\hat{f}_{t_{1}, t_{2}}(x, y, z)\right|(1+|x|)^{1 / 4} \log (2+|x|) d x d y d z \ll 1
$$

which gives us the bound

$$
\int_{\substack{x, y, z \\|x| \geq H}}\left|\hat{f}_{t_{1}, t_{2}}(x, y, z)\right| \log (2+|x|) d x d y d z \ll 1 / H^{1 / 4}
$$


The same bound holds true with the condition $|x| \geq H$ replaced by $|y| \geq H$ or $|z| \geq H$. We thus get

$$
\begin{aligned}
& \int_{x, y, z} \hat{f}_{t_{1}, t_{2}, \kappa}(x, y, z)\left(E_{0}(\varepsilon, \tau, x)-E_{0}(0, \tau, x)\right) \mathscr{E}_{\ell-1, m, n}(\varepsilon, \tau, x, y, z) d x d y d z \\
& \ll \int_{\substack{x, y, z \\
|x| \leq H}}+\int_{\substack{x, y, z,|x| \geq H}} \ldots \\
& \ll \int_{\substack{x, y, z \\
|x| \leq H}} \varepsilon H^{3 / 4}|x|^{1 / 4}\left|\hat{f}_{t_{1}, t_{2}, \kappa}(x, y, z) \mathscr{E}_{\ell-1, m, n}(\varepsilon, \tau, x, y, z)\right| d x d y d z \\
&+\int_{\substack{x, y, z \\
|x| \geq H}} \log (2+|x|)\left|\hat{f}_{t_{1}, t_{2}, \kappa}(x, y, z) \mathscr{E}_{\ell-1, m, n}(\varepsilon, \tau, x, y, z)\right| d x d y d z \\
& \ll \varepsilon H^{3 / 4}+H^{-1 / 4} .
\end{aligned}
$$

We select $H=1 / \varepsilon$ and repeat the argument for each factor. This leads to

$$
\begin{aligned}
& \mathfrak{G}_{\kappa, r}\left(t_{1}, t_{2}, \varepsilon, \tau\right)=\int_{x, y, z} d x d y d z \hat{f}_{t_{1}, t_{2}, \kappa}(x, y, z) \sum_{a+b+c+d=r} \frac{(-1)^{b}}{a ! b ! c ! d !} \sum_{\ell, m, n \geq 0} \\
&\left(\begin{array}{l}
b \\
\ell
\end{array}\right)\left(\begin{array}{c}
c \\
m
\end{array}\right)\left(\begin{array}{l}
d \\
n
\end{array}\right)\left(\log \frac{\tau}{\varepsilon}\right)^{r-\ell-m-n} \mathscr{E}_{\ell, m, n}(0, \tau, x, y, z)+\mathcal{O}\left((\log \varepsilon)^{r} \varepsilon^{1 / 4}\right) .
\end{aligned}
$$

There cannot be any dependence of the main term in $\varepsilon$. It means that each coefficient of $\log (\tau / \varepsilon)^{d}$ for positive $d$ has to vanish. This is not so obvious because of two obstructions: $P_{0}$ has to be somewhat large but restricted in size with respect to $P$; furthermore, the limit has to be taken in some $\mathrm{L}^{p}$-sense. We treat both problems one after another in the next section.

\section{A divertimento}

We have found that

$$
\begin{aligned}
\int_{0 \leq t_{1}, t_{2} \leq 1} w^{\prime}\left(t_{1}\right) w^{\prime}\left(t_{2}\right) \mathfrak{G}_{\kappa, r}\left(t_{1}, t_{2}, \varepsilon, \tau\right) d t_{1} d t_{2} & =\frac{S^{(4)} Z}{(-1)^{r} A} \\
& +\mathcal{O}_{B}\left(\frac{(\log \log P)^{r}}{R^{B}}+\frac{\log (\tau / \varepsilon)^{2 r}}{P_{0}}+\alpha^{2}(\log \log P)^{r}\left(\log P_{0}\right)^{2}+\frac{1}{R}\right) .
\end{aligned}
$$

Let us constrain $\tau$ to be bounded: $1 \leq \tau \leq T$. We bound above $1 / P_{0}$ by $1 / R^{B}$, and $H$ by $\log P$, then replace $\log P_{0}$ by $\varepsilon \log Q$. We set $q^{\prime}=\log Q$, select $B=1$ and get that the error term above is

$$
\ll_{T} \frac{\left(\log q^{\prime}\right)^{r}}{\varepsilon q^{\prime}}+\varepsilon^{2}\left(\log q^{\prime}\right)^{r}+\frac{1}{\varepsilon q^{\prime}} \ll_{T} \frac{1}{\varepsilon q}+\varepsilon^{2}(\log q)^{r} \ll_{T} \frac{1}{\varepsilon q}+\varepsilon^{2} q
$$

with $q=q^{\prime} /\left(\log q^{\prime}\right)^{r}$. We have thus at our disposal two functions $f$ and $g$ such that

$$
|f(\varepsilon)-g(q)| \ll \frac{1}{\varepsilon q}+\varepsilon^{2} q
$$

when $q$ goes to infinity and $\varepsilon$ goes to 0 . We infer below from this hypothesis that $f$ has a limit when $\varepsilon$ goes to 0 .

LEMma 13.1. Let $\delta>0$. When $1 / \delta \leq \varepsilon q \leq \delta / \varepsilon$, we have

$$
\frac{1}{\varepsilon q}+\varepsilon^{2} q \leq 2 \delta
$$


Proof. Set $x=\varepsilon q$. The last inequality is verified if and only if

$$
1-2 \delta x+\varepsilon x^{2} \leq 0
$$

The discriminant of this trinomial is $4 \delta^{2}-4 \varepsilon \geq 0$, for otherwise the interval we consider is empty. The variable $x$ has to lie between the two roots:

$$
\frac{\delta-\sqrt{\delta^{2}-\varepsilon}}{\varepsilon}=\frac{1}{\delta+\sqrt{\delta^{2}-\varepsilon}} \leq \frac{1}{\delta} \quad \text { and } \quad \frac{\delta}{\varepsilon} \leq \frac{\delta+\sqrt{\delta^{2}-\varepsilon}}{\varepsilon} .
$$

The Lemma follows readily.

LEMMA 13.2. When $\varepsilon^{2} / \delta^{2} \leq \varepsilon^{\prime} \leq \varepsilon \leq 1$, we have $\left|f(\varepsilon)-f\left(\varepsilon^{\prime}\right)\right| \leq 4 \delta$.

Proof. Let $q=\delta / \varepsilon^{2}$. We have $|f(\varepsilon)-g(q)| \leq 2 \delta$. On another hand, $1 / \delta \leq \varepsilon^{\prime} q \leq \delta / \varepsilon^{\prime}$, and thus $\left|f\left(\varepsilon^{\prime}\right)-g(q)\right| \leq 2 \delta$. The triangle inequality concludes.

We select $\delta=2 \sqrt{\varepsilon}$. Let $\left.\left.\varepsilon^{\prime} \in\right] 0, \varepsilon\right]$. There exists a non-negative integer $k$ such that $4^{-k-1} \varepsilon<$ $\varepsilon^{\prime} \leq 4^{-k} \varepsilon$. We write the difference $f\left(\varepsilon^{\prime}\right)-f(\varepsilon)$ as

$$
f\left(\varepsilon^{\prime}\right)-f\left(4^{-k} \varepsilon\right)+f\left(4^{-(k-1)} \varepsilon\right)-f\left(4^{-(k-2)} \varepsilon\right)+\cdots+f(\varepsilon / 4)-f(\varepsilon) .
$$

We can thus bound the difference by $8\left(2^{-k}+\cdots+1\right) \sqrt{\varepsilon} \leq 16 \sqrt{\varepsilon}$. The function $f$ verifies Cauchy's criterion in the neighborhood of 0 and thus admits a limit there, as wanted.

As a conclusion, we can restrict the summation in (12.7) to $r+c-\ell-m-n=0$. This means $a=0, \ell=b, m=c$ and $n=d$, and thus we have

$$
\begin{aligned}
\int_{0 \leq t_{1}, t_{2} \leq 1} w^{\prime}\left(t_{1}\right) w^{\prime}\left(t_{2}\right) \mathfrak{G}_{\kappa, r}\left(t_{1}, t_{2}, \varepsilon, \tau\right) d t_{1} d t_{2} & =\int_{0 \leq t_{1}, t_{2} \leq 1} w^{\prime}\left(t_{1}\right) w^{\prime}\left(t_{2}\right) \int_{x, y, z} d x d y d z \\
\hat{f}_{t_{1}, t_{2}, \kappa}(x, y, z) & \sum_{b+c+d=r} \frac{(-1)^{b}}{b ! c ! d !} \mathscr{E}_{b, c, d}(0, \tau, x, y, z)+\mathcal{O}\left((\log \varepsilon)^{r} \varepsilon^{1 / 4}\right) .
\end{aligned}
$$

This equality is valid for every $w^{\prime}$ bounded, and say continuous (measurable would do). We have assumed $w^{\prime}$ to be non-positive only to have an easy bound on $\lambda_{d}$, but this hypothesis is otherwise irrelevant. By polarization, we extend it to $w_{1}^{\prime}\left(t_{1}\right) w_{2}^{\prime}\left(t_{2}\right)$ for any two distinct functions $w_{1}^{\prime}$ and $w_{2}^{\prime}$. The relation holds also for linear combinations of such (elementary tensor) products, which happen to be dense in $\mathrm{L}^{2}\left([0,1]^{2}\right)$. Indeed each monomial is an elementary tensor product and Weierstrass approximation Theorem applies; this enables us to extend our relation from $w_{1}^{\prime} \otimes w_{2}^{\prime}$ to any continuous function over $[0,1]^{2}$. Such functions are dense in any $\mathrm{L}^{p}$ for $1 \leq p \leq \infty$ and this is enough to show that

$$
\begin{aligned}
\mathfrak{G}_{\kappa, r}\left(t_{1}, t_{2}, \epsilon, \tau\right)+\mathcal{O}\left((\log \varepsilon)^{r} \varepsilon^{1 / 4}\right) & = \\
& \quad \int_{x, y, z} \hat{f}_{t_{1}, t_{2}, \kappa}(x, y, z) \sum_{b+c+d=r} \frac{(-1)^{c+d}}{b ! c ! d !} \mathscr{E}_{b, c, d}(0, \tau, x, y, z) d x d y d z .
\end{aligned}
$$

We have finally reached the existence of the following function:

$$
\begin{aligned}
\mathfrak{G}_{\kappa, r}\left(t_{1}, t_{2}, \tau\right)=\lim _{\epsilon \rightarrow 0} \mathfrak{G}_{\kappa, r}\left(t_{1}, t_{2}, \epsilon, \tau\right)= \\
\qquad \int_{x, y, z} \hat{f}_{t_{1}, t_{2}, \kappa}(x, y, z) \sum_{b+c+d=r} \frac{(-1)^{c+d}}{b ! c ! d !} \mathscr{E}_{b, c, d}(0, \tau, x, y, z) d x d y d z .
\end{aligned}
$$

This expression has also the following consequences: 
LEMma 13.3. The function $\mathfrak{G}_{\kappa, r}\left(t_{1}, t_{2}, \tau\right)$ is symmetrical in $t_{1}$ and $t_{2}$. Moreover, we have $\mathfrak{G}_{\kappa, r}\left(0, t_{2}, \tau\right)=0$.

14. A general formula for weights carried on integers with $r$ prime factors

We can take the limit as $\varepsilon$ goes to 0 in(13.3) and eliminate the Fourier transform, getting

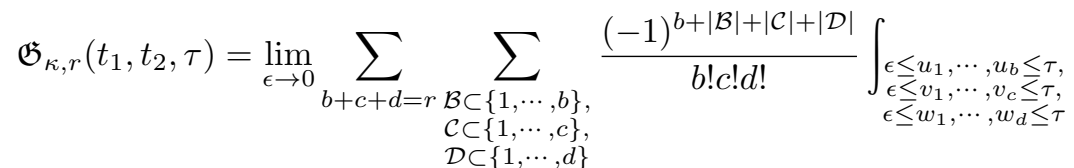

$$
\begin{aligned}
& \frac{\left(\min \left(t_{1}-\sum_{i \in \mathcal{C}} v_{i}, t_{2}-\sum_{i \in \mathcal{D}} w_{i}\right)-\sum_{i \in \mathcal{B}} u_{i}\right)^{+\kappa} \prod_{i} d u_{i} \prod_{i} d v_{i} \prod_{i} d w_{i}}{\prod_{i} u_{i} \prod_{i} v_{i} \prod_{i} w_{i}} .
\end{aligned}
$$

This formula is explicit. We can also take the limit as $\epsilon$ goes to 0 in (13.3) (replace $\varepsilon$ by $\epsilon$ ), or, after the same manipulation, in (10.4). It also reveals the following property:

LEMMA 14.1. We have $\mathfrak{G}_{\kappa, r}\left(t_{1}, t_{2}, \tau\right)=\tau^{\kappa} \mathfrak{G}_{\kappa, r}\left(t_{1} / \tau, t_{2} / \tau, 1\right)$

This leads to the main Theorem, namely

Theorem 14.1. We have, when $P=Q^{\tau}$, and with $S_{0}$ being defined in (3.2),

$$
\begin{aligned}
& (\log Q)^{\kappa} S_{0}\left(a_{r}[P] ; \mathcal{K}, \mathcal{K}^{*}\right)= \\
& A \kappa^{\prime r} \int_{0 \leq t_{1}, t_{2} \leq 1} w^{\prime}\left(t_{1}\right) w^{\prime}\left(t_{2}\right) \mathfrak{G}_{\kappa, r}\left(t_{1}, t_{2}, \tau\right) d t_{1} d t_{2}+\mathcal{O}\left(\left\|w^{\prime}\right\|_{\infty}^{2}(\log Q)^{-1 / 9}\right)
\end{aligned}
$$

where $\mathfrak{G}_{\kappa, r}$ is the bounded continuous function given above.

The error term $\mathcal{O}\left((\log Q)^{-1 / 9}\right)$ is achieved by selecting $\log P_{0}=\sqrt{\log Q}$. It is uniform in $\tau$ between 1 and some fixed bound, say $T$ (the error term depending on $T$ ). It depends on $\mathcal{K}$ and on $\mathcal{K}^{*}$. We have not tried to optimize the exponent $1 / 9$.

Conjecture 1. The function $\mathfrak{G}_{\kappa, r}$ is twice continuously differentiable in the domain $0 \leq$ $t_{1} \leq t_{2} \leq \tau$.

We can split the domain of integration in (14.1), once $\mathcal{B}, \mathcal{C}$ and $\mathcal{D}$ are fixed, into subdomains in which the expression $\left(\min \left(t_{1}-\sum_{j \in \mathcal{C}} v_{j}, t_{2}-\sum_{k \in \mathcal{D}} w_{k}\right)-\sum_{i \in \mathcal{B}} u_{i}\right)^{+\kappa}$ reduces to a polynomial. This implies conditions like

$$
\sum_{i \in \mathcal{B}} u_{i} \leq t_{2}-\sum_{k \in \mathcal{D}} w_{k} \leq t_{1}-\sum_{j \in \mathcal{C}} v_{j}
$$

In front of such a condition, we would fix the $u_{i}$ 's when $i \in \mathcal{B}$, then the $w_{k}$ 's when $k \in \mathcal{D}$ and finally the $v_{j}$ 's when $j \in \mathcal{C}$. The remaining variables are in fact independent. We would then (try to) resolve each singularity by using Lemma 20.1. This is the process we will follow when $r=0,1$ and 2 . If this program carries through, then the conjecture would be true. But we are not sure additional conditions would not pop in. Note that a discussion as to whether $t_{1} \leq t_{2}$ comes in immediately when $\mathcal{D}=\mathcal{C}=\emptyset$. It is also necessary to handle the second condition 
above:

$$
t_{2}-t_{1}+\sum_{j \in \mathcal{C}} v_{j} \leq-\sum_{k \in \mathcal{D}} w_{k}
$$

When $t_{2}-t_{1}<0$, this condition would compete with the non-negativity conditions on the $w_{k}$ 's. This discussion justifies the conjecture.

Note that the expression (14.1) implies that this function is twice continuously differentiable per pieces. Assuming this conjecture and recalling that $\mathfrak{G}_{\kappa, r}\left(0, t_{2}, \tau\right)$ vanishes, we find that

$$
\begin{aligned}
\int_{0 \leq t_{1} \leq t_{2} \leq 1} w^{\prime}\left(t_{1}\right) & w^{\prime}\left(t_{2}\right) \mathfrak{G}_{\kappa, r}\left(t_{1}, t_{2}, \tau\right) d t_{1} d t_{2} \\
=\int_{0}^{1} w^{\prime}\left(t_{2}\right)\left[0+\int_{0}^{t_{2}}(\right. & \left.\left.w\left(t_{2}\right)-w\left(t_{1}\right)\right) \frac{d \mathfrak{G}_{\kappa, r}\left(t_{1}, t_{2}, \tau\right)}{d t_{1}} d t_{1}\right] d t_{2} \\
& =\int_{0}^{1} w^{\prime}\left(t_{2}\right) \int_{0}^{t_{2}}\left(w\left(t_{2}\right)-w\left(t_{1}\right)\right) \frac{d \mathfrak{G}_{\kappa, r}\left(t_{1}, t_{2}, \tau\right)}{d t_{1}} d t_{1} d t_{2} .
\end{aligned}
$$

We use a second integration by parts, with respect to $t_{2}$, and integrate the factor $w^{\prime}\left(t_{2}\right)\left(w\left(t_{2}\right)-\right.$ $\left.w\left(t_{1}\right)\right)$ as $\frac{1}{2}\left(w\left(t_{2}\right)-w\left(t_{1}\right)\right)^{2}$. On using the facts that $w(1)=0$ we reach this way a main formula:

$$
\begin{aligned}
\int_{0 \leq t_{1}, t_{2} \leq 1} w^{\prime}\left(t_{1}\right) w^{\prime}\left(t_{2}\right) \mathfrak{G}_{\kappa, r}\left(t_{1}, t_{2}, \tau\right) d t_{1} d t_{2} \\
=\int_{0}^{1} w\left(t_{1}\right)^{2} \frac{d \mathfrak{G}_{\kappa, r}\left(t_{1}, 1, \tau\right)}{d t_{1}} d t_{1} \\
\quad-\frac{1}{2} \int_{0 \leq t_{1}, t_{2} \leq 1}\left(w\left(t_{2}\right)-w\left(t_{1}\right)\right)^{2} \frac{d^{2} \mathfrak{G}_{\kappa, r}\left(t_{1}, t_{2}, \tau\right)}{d t_{1} d t_{2}} d t_{1} d t_{2}
\end{aligned}
$$

\section{Proof of Theorem 1.2}

We restrict our attention to $\mathcal{K}=\mathcal{K}\left(h_{1}, \cdots, h_{\kappa}\right)$. As already noted at the end of section 2 , the constants $A$ and $\mathscr{C}$ are then equal and strictly positive. We select, for each $i$ in $\{1, \cdots, \kappa\}$, the additional compact set $\mathcal{K}^{*}=\mathcal{U}-h_{i}$, so that $\kappa^{\prime}=1$. Theorem 1.2 is thus simply a rewriting in this special case of Theorem 14.1 since

$$
S_{i}\left(a_{r}\left[Q^{\tau}\right]\right)=S\left(a_{r}\left[Q^{\tau}\right] ; \mathcal{K}\left(h_{1}, \cdots, h_{\kappa}\right), \mathcal{U}-h_{i}\right) .
$$

\section{On a generalisation of the Euler beta function}

We consider the family of functions defined, for every $k \geq 0$ and every real number $a, b>0$, by

$$
B_{k}(a, b)=(-1)^{k} \int_{0}^{1} t^{a-1}(1-t)^{b-1} \log ^{k}(1-t) d t \geq 0 .
$$

We will need to evaluate this function for $k=1$ and $k=2$ but it is easier to take a general path. When $k=0$, we have

$$
B(a, b)=B_{0}(a, b)=\frac{\Gamma(a) \Gamma(b)}{\Gamma(a+b)} .
$$

We notice that for non-negative $z<b$, by the Lebesgue dominated convergence Theorem, we have

$$
\sum_{k \geq 0} \frac{B_{k}(a, b) z^{k}}{k !}=\int_{0}^{1} t^{a-1}(1-t)^{b-1} \sum_{k \geq 0} \frac{(-z \log (1-t))^{k}}{k !} d t=B(a, b-z) .
$$


As a consequence, we find that:

LEMMA 15.1 .

$$
B_{k}(a, b)=\left.\frac{d^{k}}{d z^{k}} B(a, b-z)\right|_{z=0}
$$

We recall the definition of the digamma function:

$$
\psi(x)=\Gamma^{\prime}(x) / \Gamma(x) .
$$

We recall the following Lemma that we take from $[\mathbf{1}, 6.3 .2,6.4 .2,6.4 .3]$ :

LEMMA 15.2. When $m \geq 1$ and $n \geq 1$ are integers, we have

$$
\psi^{(m)}(n)=(-1)^{m+1} m !\left(-\zeta(m+1)+\sum_{1 \leq c \leq n-1} \frac{1}{c^{m+1}}\right) .
$$

In case $m=0$, the same formula holds but with $-\gamma$ instead of $-\zeta(1)$.

Lemma 15.1 leads to the following recursive formula:

LEMma 15.3. When $k \geq 0$, we have

$$
B_{k+1}(a, b)=\sum_{0 \leq \leq m \leq k}\left(\begin{array}{c}
k \\
m
\end{array}\right) B_{k-m}(a, b)(-1)^{m+1}\left(\psi^{(m)}(a+b)-\psi^{(m)}(b)\right) .
$$

Proof. We appeal to Lemma 15.1 to infer that

$$
\frac{B_{1}(a, b-z)}{B(a, b-z)}=\psi(a+b-z)-\psi(b+z) .
$$

We then use Leibniz law concerning the $k$-th derivative of a product and evaluate the resulting expression at $z=0$ to get our Lemma.

As a consequence, we find that

LEMmA 15.4. When $a$ and $b$ are positive integers, we have

$$
B_{1}(a, b)=\left(\mathcal{H}_{a+b-1}-\mathcal{H}_{b-1}\right) B(a, b) .
$$

LEMMA 15.5. When $a$ and $b$ are positive integers, we have

$$
B_{2}(a, b)=\left(\left(\mathcal{H}_{a+b-1}-\mathcal{H}_{b-1}\right)^{2}+\sum_{b \leq c \leq a+b-1} \frac{1}{c^{2}}\right) B(a, b)
$$

Proof. $\quad$ We have by Lemma 15.3

$$
B_{2}(a, b)=B_{1}(a, b)\left(\mathcal{H}_{a+b-1}-\mathcal{H}_{b-1}\right)+B(a, b) \sum_{b \leq c \leq a+b-1} \frac{1}{c^{2}}
$$

and the Lemma follows readily. 


\section{Derivatives with respect to $\tau$}

On using (10.4) and (10.2), we readily find that

$$
\begin{aligned}
\tau \frac{d \mathfrak{G}_{\kappa, r}\left(t_{1}, t_{2}, \epsilon, \tau\right)}{d \tau}=\mathfrak{G}_{\kappa, r-1}\left(t_{1},\right. & \left.t_{2}, \epsilon, \tau\right)-\mathfrak{G}_{\kappa, r-1}\left(t_{1}-\tau, t_{2}, \epsilon, \tau\right) \\
& -\mathfrak{G}_{\kappa, r-1}\left(t_{1}, t_{2}-\tau, \epsilon, \tau\right)+\mathfrak{G}_{\kappa, r-1}\left(t_{1}-\tau, t_{2}-\tau, \epsilon, \tau\right)
\end{aligned}
$$

and the same holds with $\epsilon=0$ which is (1.3). This gives us the following formula

$$
\begin{gathered}
\int_{t_{1}, t_{2}} w^{\prime}\left(t_{1}\right) w^{\prime}\left(t_{2}\right) \frac{d \mathfrak{G}_{\kappa, r}\left(t_{1}, t_{2}, \tau\right)}{d \tau} d t_{1} d t_{2}= \\
\int_{t_{1}, t_{2}}\left(w^{\prime}\left(t_{1}\right)-w^{\prime}\left(t_{1}+\tau\right)\right)\left(w^{\prime}\left(t_{2}\right)-w^{\prime}\left(t_{2}+\tau\right)\right) \frac{\mathfrak{G}_{\kappa, r-1}\left(t_{1}, t_{2}, \tau\right)}{\tau} d t_{1} d t_{2} .
\end{gathered}
$$

We can then apply (14.2) or (18.2) when $r-1=1$. There are two ways to introduce the derivative with respect to $\kappa$. The first one, that initiated this line of enquiries, consists in adding a differentiable weight $W\left(\log \max \left(p_{1}, \cdots, p_{r}\right)\right)$ to $a_{r}[P]$. We then see that we can let this weight approximate the step function $\mathbb{1}_{x \leq \log P}$. There is however a much simpler way by directly noticing that

$$
\mathfrak{G}_{\kappa, r}\left(t_{1}, t_{2}, \tau_{0}\right)=\int_{0}^{\tau_{0}} \frac{d \mathfrak{G}_{\kappa, r}\left(t_{1}, t_{2}, \tau\right)}{d \tau}
$$

since $\mathfrak{G}_{\kappa, r}\left(t_{1}, t_{2}, 0\right)=0$.

17. Special case $r=0$

We use (10.3) together with (10.4) and (10.2) and get

$$
\mathfrak{G}_{\kappa, 0}\left(t_{1}, t_{2}, \tau\right)=\min \left(t_{1}, t_{2}\right)^{\kappa} .
$$

We find that

$$
\int_{0 \leq t_{1}, t_{2} \leq 1} w^{\prime}\left(t_{1}\right) w^{\prime}\left(t_{2}\right) \mathfrak{G}_{\kappa, 0}\left(t_{1}, t_{2}, \tau\right) d t_{1} d t_{2}=\kappa \int_{0}^{1} w(t)^{2} t^{\kappa-1} d t .
$$

Proof. The following lines are straightforward:

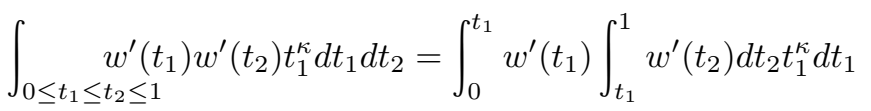

$$
\begin{aligned}
& =-\int_{0}^{t_{1}} w^{\prime}\left(t_{1}\right) w\left(t_{1}\right) t_{1}^{\kappa} d t_{1}=\frac{\kappa}{2} \int_{0}^{t_{1}} w\left(t_{1}\right)^{2} t_{1}^{\kappa-1} d t_{1}
\end{aligned}
$$

as required.

\section{Special case $r=1$}

A general expression We again use (10.3) together with (10.4) and (10.2) and get, when $t_{1} \leq t_{2}$,

$$
\mathfrak{G}_{\kappa, 1}\left(t_{1}, t_{2}, \varepsilon, \tau\right)=t_{1}^{\kappa} \log (\tau / \varepsilon)-\int_{\varepsilon}^{\tau} \frac{\max \left(0, \min \left(t_{1}, t_{2}-w\right)\right)^{\kappa} d w}{w}
$$


On discussing on the size of $\tau$ and letting $\varepsilon$ go to zero, we get the following expression:

$$
\mathfrak{G}_{\kappa, 1}\left(t_{1}, t_{2}, \tau\right)=t_{1}^{\kappa} \log \frac{\tau}{t_{2}-t_{1}}-\int_{t_{2}-t_{1}}^{\max \left(\tau, t_{2}\right)} \frac{\left(t_{2}-w\right)^{\kappa} d w}{w}
$$

where the integral is not taken in the algebraical sense, i.e. it vanishes when $\tau<t_{2}-t_{1}$.

An integral form for the associated distribution: We can eliminate $\mathfrak{G}_{\kappa, 1}$ altogether from our expressions by appealing to (14.2):

$$
\begin{aligned}
\int_{0 \leq t_{1}, t_{2} \leq 1} w^{\prime}\left(t_{1}\right) w^{\prime}\left(t_{2}\right) & \mathfrak{G}_{\kappa, 1}\left(t_{1}, t_{2}, \tau\right) d t_{1} d t_{2} / \kappa= \\
& \int_{\substack{0 \leq t<1, 1-t \leq \tau}} w(t)^{2} t^{\kappa-1} \log \frac{\tau}{1-t} d t \\
& +\int_{\substack{0 \leq t_{1}<t_{2} \leq 1, t_{2}-t_{1} \leq \tau}} \frac{\left(w\left(t_{2}\right)-w\left(t_{1}\right)\right)^{2}}{t_{2}-t_{1}} t_{1}^{\kappa-1} d t_{1} d t_{2} .
\end{aligned}
$$

Note that the right-hand side is a sum of two non-negative terms, so that no cancellations between them may occur. Note further that we have added the condition $t_{1}<t_{2}$ on the righthand side while no such condition appears on the left-hand one.

Proof. We use the (18.1) and readily get

$$
\frac{d \mathfrak{G}_{\kappa, 1}\left(t_{1}, 1, \tau\right)}{d t_{1}}=\kappa t_{1}^{\kappa-1} \log \frac{\tau}{1-t_{1}},
$$

as well as

$$
\frac{d^{2} \mathfrak{G}_{\kappa, 1}\left(t_{1}, 1, \tau\right)}{d t_{1} d t_{2}}=\frac{\kappa t_{1}^{\kappa-1}}{t_{2}-t_{1}}
$$

when $t_{2}-t_{1} \leq \tau$ and vanishes otherwise.

\section{Special case $r=2$}

An integral form for the associated distribution: We first note that, when $\tau_{0}>0$ :

$$
\begin{aligned}
\int_{0 \leq t_{1}, t_{2} \leq 1} w^{\prime}\left(t_{1}\right) w^{\prime}\left(t_{2}\right) \mathfrak{G}_{\kappa, 2}\left(t_{1}, t_{2}, \tau_{0}\right) d & t_{1} d t_{2} \\
= & \int_{0}^{\tau_{0}} \int_{0 \leq t_{1}, t_{2} \leq 1} w^{\prime}\left(t_{1}\right) w^{\prime}\left(t_{2}\right) \frac{d \mathfrak{G}_{\kappa, 2}\left(t_{1}, t_{2}, \tau\right)}{d \tau} d t_{1} d t_{2} d \tau
\end{aligned}
$$

At this stage, we employ (16.2):

$$
\begin{aligned}
\int_{0 \leq t_{1}, t_{2} \leq 1} & w^{\prime}\left(t_{1}\right) w^{\prime}\left(t_{2}\right) \mathfrak{G}_{\kappa, 2}\left(t_{1}, t_{2}, \tau_{0}\right) d t_{1} d t_{2} \\
& =\int_{0}^{\tau_{0}} \int_{0 \leq t_{1}, t_{2} \leq 1}\left(w^{\prime}\left(t_{1}\right)-w^{\prime}\left(t_{1}+\tau\right)\right)\left(w^{\prime}\left(t_{2}\right)-w^{\prime}\left(t_{2}+\tau\right)\right) \mathfrak{G}_{\kappa, 1}\left(t_{1}, t_{2}, \tau\right) d t_{1} d t_{2} \frac{d \tau}{\tau} .
\end{aligned}
$$


We then use (18.2) and reach

$$
\begin{aligned}
& \int_{0 \leq t_{1}, t_{2} \leq 1} w^{\prime}\left(t_{1}\right) w^{\prime}\left(t_{2}\right) \mathfrak{G}_{\kappa, 2}\left(t_{1}, t_{2}, \tau_{0}\right) d t_{1} d t_{2} / \kappa= \\
& \quad \int_{0}^{\tau_{0}} \int_{\substack{0 \leq t<1, 1-t \leq \tau}}(w(t)-w(t+\tau))^{2} t^{\kappa-1} \log \frac{\tau}{1-t} d t \frac{d \tau}{\tau} \\
& \quad+\int_{0}^{\tau_{0}} \int_{\substack{0 \leq t_{1}<t_{2} \leq 1, t_{2}-t_{1} \leq \tau}} \frac{\left(w\left(t_{2}\right)-w\left(t_{2}+\tau\right)-w\left(t_{1}\right)+w\left(t_{1}+\tau\right)\right)^{2}}{t_{2}-t_{1}} t_{1}^{\kappa-1} d t_{1} d t_{2} \frac{d \tau}{\tau}
\end{aligned}
$$

\section{A useful Lemma}

Here is a trivial Lemma that we will use over and over again:

LEMMA 20.1. We have

$$
\frac{(x-y)^{\kappa}-x^{\kappa}}{y}=-\sum_{1 \leq \ell \leq \kappa}(x-y)^{\kappa-\ell} x^{\ell-1} .
$$

This Lemma is one of the reasons why we have to assume $\kappa$ to be an integer. When $r$ is 0,1 or 2 , this is the sole reason. When $r$ is arbitrary, we represent in section 12 a function depending on $\kappa$ as a convolution product of $\kappa$ factors, and this again requires $\kappa$ to be an integer.

Proof. Indeed, we first note that

$$
\sum_{1 \leq \ell \leq \kappa} u^{\kappa-\ell+1} v^{\ell-1}=u^{\kappa} \frac{1-(v / u)^{\kappa}}{1-(v / u)}=u \frac{u^{\kappa}-v^{\kappa}}{u-v}
$$

where we select $u=x-y$ and $v=y$.

$$
\text { 21. Special case } w(t)=(1-t)^{+\nu} \text { and } r \leq 2
$$

We select here $w(t)=(1-t)$ and compute the main terms when $r=0,1$ and $r=2$. Let us start with a simple consequence of (17.2).

LEMMA 21.1. When $w(t)=(1-t)^{+\nu}$ with $\nu \geq 1$, and $\tau>0$, we have

$$
\nu^{2} \int_{0 \leq t_{1}, t_{2} \leq 1}\left(1-t_{1}\right)^{\nu-1}\left(1-t_{2}\right)^{\nu-1} \frac{\mathfrak{G}_{\kappa, 0}\left(t_{1}, t_{2}, \tau\right)}{\kappa} d t_{1} d t_{2}=B(2 \nu+1, \kappa) .
$$

In case $r=1$, we have formula (18.2).

LEMMA 21.2. When $w(t)=(1-t)^{+\nu}$ and $\tau \geq 1$, we have

$$
\begin{aligned}
\nu^{2} \int_{0 \leq t_{1}, t_{2} \leq 1}\left(1-t_{1}\right)^{\nu-1}\left(1-t_{2}\right)^{\nu-1} \frac{\mathfrak{G}_{\kappa, 1}\left(t_{1}, t_{2}, \tau\right)}{\kappa} d t_{1} d t_{2} & \\
= & \left(\mathcal{H}_{\kappa+2 \nu}-2 \mathcal{H}_{2 \nu}+2 \mathcal{H}_{\nu}\right) B(2 \nu+1, \kappa) .
\end{aligned}
$$


Proof. Let us call momentarily $I$ the integral to compute. We start with (18.2), getting:

$$
I=B_{1}(\kappa, 2 \nu-1)+\int_{0 \leq t_{1} \leq t_{2} \leq 1} \frac{\left(\left(1-t_{1}\right)^{\nu}-\left(1-t_{2}\right)^{\nu}\right)^{2}}{t_{2}-t_{1}} t_{1}^{\kappa-1} d t_{2} d t_{1} .
$$

We then expand one $\left(1-t_{1}\right)^{\nu}-\left(1-t_{2}\right)^{\nu}$ via Lemma 20.1:

$$
\begin{aligned}
I= & B_{1}(\kappa, 2 \nu+1)+\sum_{1 \leq k \leq \nu} \int_{0 \leq t_{1} \leq t_{2} \leq 1}\left(1-t_{1}\right)^{2 \nu-k}\left(1-t_{2}\right)^{k-1} t_{1}^{\kappa-1} d t_{2} d t_{1} \\
& -\sum_{1 \leq k \leq \nu} \int_{0 \leq t_{1} \leq t_{2} \leq 1}\left(1-t_{1}\right)^{\nu-k}\left(1-t_{2}\right)^{\nu+k-1} t_{1}^{\kappa-1} d t_{2} d t_{1} \\
= & \left(\mathcal{H}_{2 \nu+\kappa}-2 \mathcal{H}_{2 \nu}+2 \mathcal{H}_{\nu}\right) B(2 \nu+1, \kappa)
\end{aligned}
$$

on using Lemma 15.4. The Lemma follows readily.

We reach the main difficulty of this part, whose proof will take much of our energy. The reader will see that the proof is lengthy but essentially straightforward.

LEMMA 21.3. When $w(t)=(1-t)^{+\nu}$, we have

$$
\begin{array}{r}
\nu^{2} \int_{0 \leq t_{1}, t_{2} \leq 1}\left(1-t_{2}\right)^{\nu-1}\left(1-t_{1}\right)^{\nu-1} \frac{\mathfrak{G}_{\kappa, 2}\left(t_{1}, t_{2}, 1\right)}{\kappa \cdot B(2 \nu+1, \kappa)} d t_{1} d t_{2} \\
=\frac{1}{2} \mathcal{H}_{\kappa+2 \nu}\left(\mathcal{H}_{\kappa+2 \nu}-4 \mathcal{H}_{2 \nu}+4 \mathcal{H}_{\nu}\right)+\mathcal{O}(\log (2 \nu)) .
\end{array}
$$

The proof starts by (19.1). We then appeal to (22.1) and (22.7) that are proven in next section. We have already noted that $K(\nu)=\frac{1}{2} \log ^{2} \nu+\mathcal{O}(\log \nu)$. We shuffle the terms around and get the Lemma.

22. Proof of Lemma 21.3: usage of (19.1) when $\tau_{0}=1$ and $r=2$

We use $(19.1)$ with $\tau_{0}=1$ and $w(t)=(1-t)^{+\nu}$. In the first term, the condition $1 \leq t+\tau$ implies that $-w(t+\tau)=0$. On using Lemma 21.1 and Lemma 21.2, we find that this first term equals $\frac{1}{2} B_{2}(\kappa, 2 \nu+1)$ i.e. by Lemma 15.5 :

$$
\begin{aligned}
\int_{0}^{1} \int_{\substack{0 \leq t<1, 1-t \leq \tau}}\left((1-t)^{\nu}-(1-t-\tau)^{+\nu}\right)^{2} t^{\kappa-1} \log \frac{\tau}{1-t} d t \frac{d \tau}{\tau} \\
\quad=\frac{1}{2}\left(\left(\mathcal{H}_{2 \nu+\kappa}-\mathcal{H}_{2 \nu}\right)^{2}+\sum_{2 \nu+1 \leq c \leq 2 \nu+\kappa} \frac{1}{c^{2}}\right) B(2 \nu+1, \kappa) .
\end{aligned}
$$

Concerning the second term, there are several ranges to consider, according to the location of $1-\tau$ with respect to $t_{1}$ and $t_{2}$.

22.1. When $t_{2} \leq 1-\tau$ :

Remember that we should also have $t_{2} \leq t_{1}+\tau$. We use Lemma 20.1 and the decomposition:

$$
\begin{aligned}
\frac{\left(1-t_{2}\right)^{\nu}-\left(1-t_{2}-\tau\right)^{\nu}}{\tau}- & \frac{\left(1-t_{1}\right)^{\nu}-\left(1-t_{1}-\tau\right)^{\nu}}{\tau}= \\
& \sum_{1 \leq k \leq \nu}\left[\left(1-t_{2}\right)^{\nu-k}\left(1-t_{2}-\tau\right)^{k-1}-\left(1-t_{1}\right)^{\nu-k}\left(1-t_{1}-\tau\right)^{k-1}\right]
\end{aligned}
$$


Page 30 of 40

O. RAMARÉ

as well as

$$
\begin{aligned}
\frac{\left(1-t_{2}\right)^{\nu}-\left(1-t_{1}\right)^{\nu}}{t_{2}-t_{1}}- & \frac{\left(1-t_{2}-\tau\right)^{\nu}-\left(1-t_{1}-\tau\right)^{\nu}}{t_{2}-t_{1}}= \\
& -\sum_{1 \leq \ell \leq \nu}\left[\left(1-t_{2}\right)^{\nu-\ell}\left(1-t_{1}\right)^{\ell-1}-\left(1-t_{2}-\tau\right)^{\nu-\ell}\left(1-t_{1}-\tau\right)^{\ell-1}\right] .
\end{aligned}
$$

We multiply both and get this product to equal $-\left(A_{1}-A_{2}-A_{3}+A_{4}\right)$ where

$$
\begin{gathered}
A_{1}=\sum_{1 \leq k, \ell \leq \nu}\left(1-t_{2}\right)^{2 \nu-k-\ell}\left(1-t_{2}-\tau\right)^{k-1}\left(1-t_{1}\right)^{\ell-1}, \\
A_{2}=\sum_{1 \leq k, \ell \leq \nu}\left(1-t_{1}\right)^{\nu-k+\ell-1}\left(1-t_{1}-\tau\right)^{k-1}\left(1-t_{2}\right)^{\nu-\ell}, \\
A_{3}=\sum_{1 \leq k, \ell \leq \nu}\left(1-t_{2}\right)^{\nu-k}\left(1-t_{2}-\tau\right)^{\nu-\ell+k-1}\left(1-t_{1}-\tau\right)^{\ell-1},
\end{gathered}
$$

and

$$
A_{4}=\sum_{1 \leq k, \ell \leq \nu}\left(1-t_{1}\right)^{\nu-k}\left(1-t_{1}-\tau\right)^{k-1+\ell-1}\left(1-t_{2}-\tau\right)^{\nu-\ell}
$$

We consider each summand separately.

22.1.1. Contribution of $A_{1}$ Notice first that

$$
\int_{t_{2}-t_{1} \leq \tau \leq 1-t_{2}} A_{1} d \tau=\sum_{1 \leq k, \ell \leq \nu} \frac{1}{k}\left(1-t_{2}\right)^{2 \nu-k-\ell}\left(1-t_{1}\right)^{\ell-1}\left(1+t_{1}-2 t_{2}\right)^{k}
$$

This also means that $t_{2} \leq\left(1+t_{1}\right) / 2$, otherwise the range of integration is empty. This upper bound is always larger than $t_{1}$. We thus get

$$
\int_{t_{1}}^{\left(1+t_{1}\right) / 2} \int_{t_{2}-t_{1}}^{1-t_{2}} A_{1} d \tau d t_{2}=\sum_{1 \leq k, \ell \leq \nu} \frac{1}{k}\left(1-t_{1}\right)^{\ell-1} \int_{t_{1}}^{\left(1+t_{1}\right) / 2}\left(1-t_{2}\right)^{2 \nu-k-\ell}\left(1+t_{1}-2 t_{2}\right)^{k} d t_{2} .
$$

Concerning the inner integral, note that

$$
\begin{aligned}
& \int_{t_{1}}^{\left(1+t_{1}\right) / 2}\left(1-t_{2}\right)^{2 \nu-k-\ell}\left(1+t_{1}-2 t_{2}\right)^{k} d t_{2} \\
& =2^{k} \int_{0}^{\left(1-t_{1}\right) / 2}\left(\frac{1-t_{1}}{2}+t_{3}\right)^{2 \nu-k-\ell} t_{3}^{k} d t_{3} \\
& \quad=2^{k}\left(\frac{1-t_{1}}{2}\right)^{2 \nu-\ell+1} \int_{0}^{1}(1+t)^{2 \nu-k-\ell} t^{k} d t
\end{aligned}
$$

and this means we can write

$$
\int_{t_{1}}^{\left(1+t_{1}\right) / 2} \int_{t_{2}-t_{1}}^{1-t_{2}} A_{1} d \tau d t_{2}=K_{1}(\nu)\left(1-t_{1}\right)^{2 \nu}
$$


for some coefficient $K_{1}(\nu)$. An expression of this coefficient is obtained by setting $t_{1}=0$ :

$$
\begin{aligned}
K_{1}(\nu)=\int_{0}^{1 / 2} \int_{t_{2}}^{1-t_{2}} \sum_{1 \leq k, \ell \leq \nu}\left(1-t_{2}\right)^{2 \nu-k-\ell}\left(1-t_{2}-\tau\right)^{k-1} d \tau d t_{2} \\
=\sum_{1 \leq k, \ell \leq \nu} \frac{1}{k} \int_{0}^{1 / 2}\left(1-t_{2}\right)^{2 \nu-k-\ell}\left(1-2 t_{2}\right)^{k} d t_{2} \\
\leq \sum_{1 \leq k, \ell \leq \nu} \frac{1}{k} \int_{0}^{1 / 2}\left(1-t_{2}\right)^{2 \nu-\ell} d t_{2} \leq \mathcal{H}_{\nu}\left(\mathcal{H}_{2 \nu}-\mathcal{H}_{\nu}\right)
\end{aligned}
$$

22.1.2. Contribution of $A_{2}$ We proceed as above and first integrate in $\tau$

$$
\int_{t_{2}-t_{1} \leq \tau \leq 1-t_{2}} A_{2} d \tau=\sum_{1 \leq k, \ell \leq \nu} \frac{1}{k}\left(1-t_{1}\right)^{\nu-k+\ell-1}\left[\left(1-t_{2}\right)^{k}-\left(t_{2}-t_{1}\right)^{k}\right]\left(1-t_{2}\right)^{\nu-\ell}
$$

We next notice that

$$
\begin{aligned}
\int_{t_{1}}^{\left(1+t_{1}\right) / 2} \sum_{1 \leq k, \ell \leq \nu} & \frac{1}{k}\left(1-t_{1}\right)^{\nu-k+\ell-1}\left(1-t_{2}\right)^{\nu+k-\ell} d t_{2} \\
& =\sum_{1 \leq k, \ell \leq \nu} \frac{1}{\nu+k-\ell+1}\left(1-t_{1}\right)^{\nu-k+\ell-1}\left(1-2^{-(\nu+k-\ell+1)}\right)\left(1-t_{1}\right)^{\nu+k-\ell+1} .
\end{aligned}
$$

Concerning the other part, we first notice that

$$
\int_{t_{1}}^{1}\left(t_{2}-t_{1}\right)^{k}\left(1-t_{2}\right)^{\nu-\ell} d t_{2}=\left(1-t_{1}\right)^{\nu-\ell+k+1} B(k+1, \nu-\ell+1)
$$

As a conclusion, there exists a coefficient $K_{2}(\nu)$ such that

$$
\int_{0}^{1} \int_{t_{1}}^{\left(1+t_{1}\right) / 2} \int_{t_{2}-t_{1}}^{1-t_{2}} A_{2} d \tau d t_{2} t_{1}^{\kappa-1} d t_{1}=K_{2}(\nu) B(2 \nu+1, \kappa)
$$

We get an expression of this coefficient by specializing $t_{1}=0$ :

$$
\begin{aligned}
K_{2}(\nu)=\int_{0}^{1 / 2} \int_{t_{2}}^{1-t_{2}} \sum_{1 \leq k, \ell \leq \nu}(1-\tau)^{k-1}\left(1-t_{2}\right)^{\nu-\ell} d \tau d t_{2} \\
\quad=\int_{0}^{1 / 2} \sum_{1 \leq k, \ell \leq \nu} \frac{1}{k}\left(\left(1-t_{2}\right)^{k}-t_{2}^{k}\right)\left(1-t_{2}\right)^{\nu-\ell} d t_{2} \\
=\int_{0}^{1 / 2} \sum_{1 \leq k, \ell \leq \nu} \frac{1}{k}\left(1-t_{2}\right)^{\nu+k-\ell} d t_{2}+\mathcal{O}^{*}\left(\sum_{1 \leq k, \ell \leq \nu} \frac{1}{2^{k} k(\nu-\ell+1)}\right) \\
=\sum_{1 \leq k, \ell \leq \nu} \frac{1}{k(k+\ell)}+\mathcal{O}(\log \nu) .
\end{aligned}
$$

Furthermore

$$
\sum_{1 \leq k, \ell \leq \nu} \frac{1}{k(k+\ell)}=\sum_{1 \leq k, \ell \leq \nu} \frac{1}{\ell}\left(\frac{1}{k}-\frac{1}{k+\ell}\right)=\mathcal{H}_{\nu}^{2}-\sum_{1 \leq k, \ell \leq \nu} \frac{1}{\ell(k+\ell)}
$$

so that

$$
K_{2}(\nu)=\frac{1}{2} \mathcal{H}_{\nu}^{2}+\mathcal{O}(\log \nu)
$$


22.1.3. Contribution of $A_{3}$ The quantity $A_{3}$ is defined in (22.4). We start with an auxiliary formula:

\section{LEMMA 22.1 .}

$$
\int_{x}^{y}(1-t)^{h}(y-t)^{b} d t=\sum_{0 \leq g \leq h} \frac{(-1)^{g} h ! b !}{(b+g+1) !(h-g) !}(y-x)^{b+1+g}(1-x)^{h-g}
$$

Proof. Let us call $I(h, b)$ the integral to evaluate. The formula is correct when $h=0$. We readily get

$$
\begin{aligned}
I(h, b) & =\left[-(1-t)^{h} \frac{(y-t)^{b+1}}{b+1}\right]_{x}^{y}-\frac{h}{b+1} \int_{x}^{y}(1-t)^{h-1}(y-t)^{b+1} d t \\
& =(1-x)^{h} \frac{\left(y-t_{1}\right)^{b+1}}{b+1}-\frac{h}{b+1} I(h-1, b+1) .
\end{aligned}
$$

We use the above Lemma to get:

$$
\begin{aligned}
& \int_{t_{2}-t_{1}}^{1-t_{2}} A_{3} d \tau=\sum_{1 \leq k, \ell \leq \nu}\left(1-t_{2}\right)^{\nu-k} \int_{t_{2}-t_{1}}^{1-t_{2}}\left(1-t_{1}-\tau\right)^{\ell-1}\left(1-t_{2}-\tau\right)^{\nu-\ell+k-1} d \tau \\
& =\sum_{1 \leq k, \ell \leq \nu}\left(1-t_{2}\right)^{\nu-k} \int_{t_{2}}^{1-t_{2}+t_{1}}(1-\tau)^{\ell-1}\left(1-t_{2}+t_{1}-\tau\right)^{\nu-\ell+k-1} d \tau \\
& =\sum_{\substack{1 \leq k, \ell \leq \nu \\
0 \leq g \leq \ell-1}} \frac{(-1)^{g}(\ell-1) !(\nu-\ell+k-1) !}{(\nu-\ell+k+g) !(\ell-1-g) !}\left(1+t_{1}-2 t_{2}\right)^{\nu-\ell+k+g}\left(1-t_{2}\right)^{\nu-k+\ell-1-g} \\
& =\sum_{\substack{1 \leq k, \ell \leq \nu \\
0 \leq g \leq \ell-1}} \frac{(-1)^{g+\ell+1}(\ell-1) !(\nu-\ell+k-1) !}{(\nu-1+g+k) ! g !}\left(1+t_{1}-2 t_{2}\right)^{\nu-1-g+k}\left(1-t_{2}\right)^{\nu-k+g} .
\end{aligned}
$$

Our next step is to carry out the integration over $t_{2}$ :

$$
\begin{aligned}
& \int_{t_{1}}^{\left(1+t_{1}\right) / 2} \int_{t_{2}-t_{1}}^{1-t_{2}} A_{3} d \tau d t_{2}=\sum_{\substack{1 \leq k, \ell \leq \nu \\
0 \leq g \leq \ell-1}} \frac{(-1)^{g+\ell+1}(\ell-1) !(\nu-\ell+k-1) !}{(\nu-1+g+k+b) ! g !} \\
& \int_{t_{1}}^{\left(1+t_{1}\right) / 2}\left(1+t_{1}-2 t_{2}\right)^{\nu-1-g+k}\left(1-t_{2}\right)^{\nu-k+g} d t_{2}=K_{3}(\nu)\left(1-t_{1}\right)^{2 \nu}
\end{aligned}
$$

for some coefficient $K_{3}(\nu)$. Finally

$$
\int_{0}^{1} \int_{0}^{1-t_{1}} \int_{t_{1}}^{1-\tau} A_{3} d t_{2} d \tau t_{1}^{\kappa-1} d t_{1}=K_{3}(\nu) B(2 \nu+1, \kappa)
$$


We again recover an expression of $K_{3}(\nu)$ by specializing $t_{1}=0$ :

$$
\begin{aligned}
& K_{3}(\nu)= \int_{0}^{1 / 2} \int_{t_{2}}^{1-t_{2}} \sum_{1 \leq k, \ell \leq \nu}\left(1-t_{2}\right)^{\nu-k}\left(1-t_{2}-\tau\right)^{\nu-\ell+k-1}(1-\tau)^{\ell-1} d \tau d t_{2} \\
&=\int_{1 / 2}^{1} \int_{1-t_{2}}^{t_{2}} \sum_{1 \leq k, \ell \leq \nu} t_{2}^{\nu-k}\left(t_{2}+\tau-1\right)^{\nu-\ell+k-1} \tau^{\ell-1} d \tau d t_{2} \\
& \leq \int_{1 / 2}^{1} \int_{1-t_{2}}^{t_{2}} \sum_{1 \leq k, \ell \leq \nu} t_{2}^{2 \nu-\ell-1} \tau^{\ell-1} d \tau d t_{2} \leq \int_{1 / 2}^{1} \int_{1-t_{2}}^{t_{2}} \sum_{1 \leq k, \ell \leq \nu} t_{2}^{\nu-k} \tau^{\nu+k-2} d \tau d t_{2} \\
& \leq \mathcal{H}_{\nu}\left(\mathcal{H}_{2 \nu-1}-\mathcal{H}_{\nu-1}\right) .
\end{aligned}
$$

22.1.4. Contribution of $A_{4}$ The argument is routine now. We first note that

$$
\int_{t_{2}-t_{1}}^{1-t_{2}} A_{4} d \tau=\sum_{1 \leq k, \ell \leq \nu}\left(1-t_{1}\right)^{\nu-k} \int_{t_{2}-t_{1}}^{1-t_{2}}\left(1-t_{1}-\tau\right)^{k-1+\ell-1}\left(1-t_{2}-\tau\right)^{\nu-\ell} d \tau
$$

The next integration leads to

$$
\int_{t_{1}}^{\left(1+t_{1}\right) / 2} \int_{t_{2}-t_{1}}^{1-t_{2}} A_{4} d \tau d t_{2}=K_{4}(\nu)\left(1-t_{1}\right)^{2 \nu}
$$

with

$$
\begin{aligned}
K_{4}(\nu) & =\int_{0}^{1 / 2} \int_{t_{2}}^{1-t_{2}} \sum_{1 \leq k, \ell \leq \nu}(1-\tau)^{k+\ell-2}\left(1-t_{2}-\tau\right)^{\nu-\ell} d \tau d t_{2} \\
= & \int_{0}^{1} \sum_{1 \leq k, \ell \leq \nu} \frac{(1-\tau)^{k-1+\ell-1}}{\nu-\ell+1}\left((1-\tau)^{\nu-\ell+1}-(1-\tau-\min (\tau, 1-\tau))^{\nu-\ell+1}\right) d \tau \\
& \leq \int_{0}^{1} \sum_{1 \leq k, \ell \leq \nu} \frac{(1-\tau)^{\nu+k-1}}{\nu-\ell+1} d \tau=\mathcal{H}_{\nu}\left(\mathcal{H}_{2 \nu}-\mathcal{H}_{\nu}\right) .
\end{aligned}
$$

22.2. When $0 \leq t_{1} \leq 1-\tau \leq t_{2} \leq t_{1}+\tau$ :

These conditions implies that $t_{1} \geq \min (0,1-2 \tau)$ and thus $\tau \geq\left(1-t_{1}\right) / 2$. We use Lemma 20.1 and the decomposition:

$$
\frac{\left(1-t_{2}\right)^{\nu}-\left(1-t_{1}\right)^{\nu}+\left(1-t_{1}-\tau\right)^{\nu}}{\tau}=\frac{\left(1-t_{2}\right)^{\nu}}{\tau}-\sum_{1 \leq k \leq \nu}\left(1-t_{1}\right)^{\nu-k}\left(1-t_{1}-\tau\right)^{k-1}
$$

as well as

$$
\frac{\left(1-t_{2}\right)^{\nu}-\left(1-t_{1}\right)^{\nu}+\left(1-t_{1}-\tau\right)^{\nu}}{t_{2}-t_{1}}=\frac{\left(1-t_{1}-\tau\right)^{\nu}}{t_{2}-t_{1}}-\sum_{1 \leq \ell \leq \nu}\left(1-t_{2}\right)^{\nu-\ell}\left(1-t_{1}\right)^{\ell-1}
$$


and get that their product is equal to $C_{1}-C_{2}-C_{3}+C_{4}$, with

$$
\begin{aligned}
C_{1}= & \frac{\left(1-t_{2}\right)^{\nu}}{\tau} \frac{\left(1-t_{1}-\tau\right)^{\nu}}{t_{2}-t_{1}} \\
= & \frac{\left(1-t_{2}\right)^{\nu}}{t_{2}-t_{1}} \frac{\left(1-t_{1}\right)^{\nu}}{\tau}-\sum_{1 \leq k \leq \nu}\left(1-t_{1}\right)^{\nu-k}\left(1-t_{1}-\tau\right)^{k-1} \frac{\left(1-t_{2}\right)^{\nu}}{t_{2}-t_{1}} \\
= & \frac{\left(1-t_{1}\right)^{2 \nu}}{\tau\left(t_{2}-t_{1}\right)}-\sum_{1 \leq k \leq \nu}\left(1-t_{2}\right)^{\nu-k} \frac{\left(1-t_{1}\right)^{\nu+k-1}}{\tau} \\
& \quad-\sum_{1 \leq k \leq \nu}\left(1-t_{1}\right)^{\nu-k}\left(1-t_{1}-\tau\right)^{k-1} \frac{\left(1-t_{1}\right)^{\nu}}{t_{2}-t_{1}} \\
& \quad+\sum_{1 \leq k, \ell \leq \nu}\left(1-t_{1}\right)^{\nu-k+\ell-1}\left(1-t_{1}-\tau\right)^{k-1}\left(1-t_{2}\right)^{\nu-\ell} \\
= & C_{1,1}-C_{1,2}-C_{1,3}+C_{4},
\end{aligned}
$$

say; we further have

$$
C_{2}=\frac{\left(1-t_{2}\right)^{\nu}}{\tau} \sum_{1 \leq \ell \leq \nu}\left(1-t_{2}\right)^{\nu-\ell}\left(1-t_{1}\right)^{\ell-1}
$$

then

$$
C_{3}=\frac{\left(1-t_{1}-\tau\right)^{\nu}}{t_{2}-t_{1}} \sum_{1 \leq k \leq \nu}\left(1-t_{1}\right)^{\nu-k}\left(1-t_{1}-\tau\right)^{k-1}
$$

and finally

$$
C_{4}=\sum_{1 \leq k, \ell \leq \nu}\left(1-t_{1}\right)^{\nu-k+\ell-1}\left(1-t_{1}-\tau\right)^{k-1}\left(1-t_{2}\right)^{\nu-\ell} .
$$

22.2.1. Contribution of $C_{4}$ We first notice that

$$
\begin{aligned}
\int_{1-\tau}^{t_{1}+\tau} C_{4} d t_{2}= & \sum_{1 \leq k, \ell \leq \nu} \frac{1}{\nu-\ell+1}\left(1-t_{1}\right)^{\nu-k+\ell-1}\left(1-t_{1}-\tau\right)^{k-1} \tau^{\nu-\ell+1} \\
& \quad-\sum_{1 \leq k, \ell \leq \nu} \frac{1}{\nu-\ell+1}\left(1-t_{1}\right)^{\nu-k+\ell-1}\left(1-t_{1}-\tau\right)^{\nu-\ell+k} .
\end{aligned}
$$

Our next step is to carry out the integration over $\tau$, getting

$$
\begin{aligned}
& \int_{\left(1-t_{1}\right) / 2}^{1-t_{1}} \int_{1-\tau}^{t_{1}+\tau} C_{4} d t_{2} d \tau \\
& \int_{\left(1-t_{1}\right) / 2} \sum_{1 \leq k, \ell \leq \nu} \frac{1}{\nu-\ell+1}\left(1-t_{1}\right)^{\nu-k+\ell-1}\left(1-t_{1}-\tau\right)^{k-1} \tau^{\nu-\ell+1} d \tau \\
& \quad-\sum_{1 \leq k, \ell \leq \nu} \frac{1}{(\nu-\ell+1)(\nu-\ell+k+1)}\left(1-t_{1}\right)^{2 \nu} \\
& \quad+\sum_{1 \leq k, \ell \leq \nu} \frac{1}{(\nu-\ell+1) 2^{\nu-\ell+k+1}(\nu-\ell+k+1)}\left(1-t_{1}\right)^{2 \nu} .
\end{aligned}
$$

Concerning the inner integrals, we note that

$$
\int_{\left(1-t_{1}\right) / 2}^{1-t_{1}}\left(1-t_{1}-\tau\right)^{k-1} \tau^{\nu-\ell+1} d \tau=\left(1-t_{1}\right)^{\nu-\ell+1+k} B^{b}(k, \nu-\ell+2)
$$


with

$$
B^{b}(a, b)=\int_{1 / 2}^{1}(1-t)^{a-1} t^{b-1} d t .
$$

As a conclusion, there exists a coefficient $K_{10}(\nu)$ such that

$$
\int_{0}^{1} \int_{\left(1-t_{1}\right) / 2}^{1-t_{1}} \int_{1-\tau}^{t_{1}+\tau} C_{4} d t_{2} d \tau t_{1}^{\kappa-1} d t_{1}=K_{10}(\nu) B(2 \nu+1, \kappa) .
$$

As before, we get an integral representation of $K_{10}(\nu)$ by selecting $t_{1}=0$ :

$$
\begin{aligned}
K_{10}(\nu) & =\int_{1 / 2}^{1} \int_{1-\tau}^{\tau} \sum_{1 \leq k, \ell \leq \nu}(1-\tau)^{k-1}\left(1-t_{2}\right)^{\nu-\ell} d \tau d t_{2} \\
& =\int_{1 / 2}^{1} \sum_{1 \leq k, \ell \leq \nu}(1-\tau)^{k-1} \frac{\tau^{\ell}-(1-\tau)^{\ell}}{\ell} d \tau \\
& =\int_{1 / 2}^{1} \sum_{1 \leq \ell \leq \nu} \frac{\tau^{\ell-1}}{\ell}\left(1-(1-\tau)^{\nu}\right) d \tau-\sum_{1 \leq k, \ell \leq \nu} \frac{1}{2^{k+\ell} \ell(k+\ell)} \ll 1 .
\end{aligned}
$$

22.2.2. Contribution of $C_{1}$ We have written $C_{1}=C_{1,1}-C_{1,2}-C_{1,3}+C_{4}$ and we study each term individually. We first handle $C_{1,1}$. We notice that:

$$
\int_{1-\tau}^{t_{1}+\tau} \frac{\left(1-t_{1}\right)^{2 \nu}}{\tau\left(t_{2}-t_{1}\right)} d t_{2}=\frac{\left(1-t_{1}\right)^{2 \nu}}{\tau} \log \frac{\tau}{1-\tau-t_{1}} .
$$

Consequently, we find that

$$
\begin{aligned}
\int_{\left(1-t_{1}\right) / 2}^{1-t_{1}} \int_{1-\tau}^{t_{1}+\tau} C_{1,1} d t_{2} d \tau & =\left(1-t_{1}\right)^{2 \nu} \int_{\left(1-t_{1}\right) / 2}^{1-t_{1}} \log \frac{\frac{\tau}{1-t_{1}}}{1-\frac{\tau}{1-t_{1}}} \frac{d \tau}{\tau} \\
& =\left(1-t_{1}\right)^{2 \nu} \int_{1 / 2}^{1} \log \frac{\tau}{1-\tau} \frac{d \tau}{\tau}
\end{aligned}
$$

and thus there exists a constant $K_{5}$ such that

$$
\int_{0}^{1} \int_{\left(1-t_{1}\right) / 2}^{1-t_{1}} \int_{1-\tau}^{t_{1}+\tau} \frac{\left(1-t_{1}\right)^{2 \nu}}{\tau\left(t_{2}-t_{1}\right)} d t_{2} t_{1}^{\kappa-1} d t_{1}=K_{5} B(2 \nu+1, \kappa) .
$$

We now handle the contribution of $C_{1,2}$. We start by noticing that:

$$
\begin{aligned}
\int_{1-\tau}^{t_{1}+\tau} C_{1,2} d t_{2}= & \sum_{0 \leq k \leq \nu-1} \frac{\left(1-t_{1}-\tau\right)^{\nu-k}}{\nu-k} \frac{\left(1-t_{1}\right)^{\nu+k}}{\tau}-\sum_{1 \leq k \leq \nu} \frac{\tau^{\nu-k}}{\nu-k+1}\left(1-t_{1}\right)^{\nu+k-1} \\
= & \mathcal{H}_{\nu} \frac{\left(1-t_{1}\right)^{2 \nu}}{\tau}-\sum_{\substack{0 \leq k \leq \nu-1, 1 \leq \ell \leq \nu-k}} \frac{\left(1-t_{1}-\tau\right)^{\nu-k-\ell}}{\nu-k}\left(1-t_{1}\right)^{\nu+\ell+k-1}-\sum_{1 \leq k \leq \nu} \frac{\tau^{\nu-k}\left(1-t_{1}\right)^{\nu+k-1}}{\nu-k+1} .
\end{aligned}
$$

Hence we can write

$$
\begin{aligned}
\int_{\left(1-t_{1}\right) / 2}^{1-t_{1}} \int_{1-\tau}^{t_{1}+\tau} C_{1,2} d t_{2} d \tau= & \mathcal{H}_{\nu}\left(1-t_{1}\right)^{2 \nu} \log 2-\sum_{\substack{1 \leq k \leq \nu, 1 \leq \ell \leq \nu-k+1}} \frac{\left(1-2^{-(\nu-k+2-\ell)}\right)\left(1-t_{1}\right)^{2 \nu}}{(\nu-k+1)(\nu-k+2-\ell)} \\
& -\sum_{\substack{1 \leq k \leq \nu\\
}} \frac{1-2^{-(\nu-k+1)}}{(\nu-k+1)^{2}}\left(1-t_{1}\right)^{2 \nu} \\
= & K_{6}(\nu)\left(1-t_{1}\right)^{2 \nu}
\end{aligned}
$$


for some coefficient $K_{6}(\nu)$. We get an integral expression for $K_{6}(\nu)$ by selecting $t_{1}=0$ :

$$
K_{6}(\nu)=\int_{1 / 2}^{1} \int_{1-\tau}^{\tau} \sum_{1 \leq k \leq \nu}\left(1-t_{2}\right)^{\nu-k} d t_{2} \frac{d \tau}{\tau}=\int_{1 / 2}^{1} \sum_{1 \leq k \leq \nu} \frac{\tau^{k}-(1-\tau)^{k}}{k} \frac{d \tau}{\tau} \leq \pi^{2} / 6 .
$$

We turn our attention to $C_{1,3}$, first getting:

$$
\int_{1-\tau}^{t_{1}+\tau} C_{1,3} d t_{2}=\sum_{1 \leq k \leq \nu}\left(1-t_{1}\right)^{2 \nu-k}\left(1-t_{1}-\tau\right)^{k-1} \log \frac{\tau}{1-\tau-t_{1}} .
$$

We note that

$$
\begin{aligned}
& \int_{\left(1-t_{1}\right) / 2}^{1-t_{1}} \sum_{1 \leq k \leq \nu}\left(1-t_{1}\right)^{2 \nu-k}\left(1-t_{1}-\tau\right)^{k-1} \log \frac{\frac{\tau}{1-t_{1}}}{1-\frac{\tau}{1-t_{1}}} d \tau \\
&=\int_{1 / 2}^{1} \sum_{1 \leq k \leq \nu}(1-\tau)^{k-1} \log \frac{\tau}{1-\tau} d \tau\left(1-t_{1}\right)^{2 \nu}
\end{aligned}
$$

Furthermore, we have

$$
\begin{aligned}
& K_{7}(\nu)=\int_{1 / 2}^{1} \sum_{1 \leq k \leq \nu}(1-\tau)^{k-1} \log \frac{\tau}{1-\tau} d \tau \\
& \quad=\int_{0}^{1 / 2} \sum_{1 \leq k \leq \nu} \tau^{k-1} \log \frac{\tau}{1-\tau} d \tau=\sum_{1 \leq k \leq \nu} \frac{1}{k} \int_{0}^{1 / 2} \tau^{k}\left(\frac{1}{\tau}+\frac{1}{1-\tau}\right) d \tau \ll 1 .
\end{aligned}
$$

As a conclusion, we find that

$$
\int_{0}^{1} \int_{\left(1-t_{1}\right) / 2}^{1-t_{1}} \int_{1-\tau}^{t_{1}+\tau} C_{1,3} d t_{2} d \tau t_{1}^{\kappa-1} d t_{1}=K_{7}(\nu) B(2 \nu+1, \kappa) .
$$

22.2.3. Contribution of $C_{2}$ We handle the integration with respect to $t_{2}$ at the beginning, obtaining

$$
\begin{aligned}
\int_{1-\tau}^{t_{1}+\tau} C_{2} d t_{2}= & \sum_{1 \leq \ell \leq \nu} \frac{1}{2 \nu-\ell+1} \tau^{2 \nu-\ell}\left(1-t_{1}\right)^{\ell-1} \\
& -\sum_{1 \leq \ell \leq \nu} \frac{1}{2 \nu-\ell+1} \frac{\left(1-t_{1}-\tau\right)^{2 \nu-\ell+1}}{\tau}\left(1-t_{1}\right)^{\ell-1} \\
= & \sum_{1 \leq \ell \leq \nu} \frac{1}{2 \nu-\ell+1} \tau^{2 \nu-\ell}\left(1-t_{1}\right)^{\ell-1}-\sum_{1 \leq \ell \leq \nu} \frac{1}{2 \nu-\ell+1} \frac{\left(1-t_{1}\right)^{2 \nu}}{\tau} \\
& \quad+\sum_{\substack{1 \leq \ell \leq \nu, 1 \leq k \leq 2 \nu-\ell+1}} \frac{1}{2 \nu-\ell+1}\left(1-t_{1}-\tau\right)^{2 \nu-\ell+1-k}\left(1-t_{1}\right)^{k+\ell-2} .
\end{aligned}
$$

As before, we conclude from these lines that there exists a coefficient $K_{8}(\nu)$ such that

$$
\int_{\left(1-t_{1}\right) / 2}^{1-t_{1}} \int_{1-\tau}^{t_{1}+\tau} C_{2} d t_{2} d \tau=K_{8}(\nu)\left(1-t_{1}\right)^{2 \nu}
$$

On setting $t_{1}=0$ in the above equation, we get

$$
K_{8}(\nu)=\int_{1 / 2}^{1} \int_{1-\tau}^{\tau} \sum_{1 \leq \ell \leq \nu}\left(1-t_{2}\right)^{2 \nu-\ell} d t_{2} \frac{d \tau}{\tau}=\int_{1 / 2}^{1} \sum_{1 \leq \ell \leq \nu} \frac{(1-\tau)^{2 \nu-\ell+1}-\tau^{2 \nu-\ell+1}}{2 \nu-\ell+1} \frac{d \tau}{\tau} \ll 1 .
$$


As a conclusion, we find that

$$
\int_{0}^{1} \int_{\left(1-t_{1}\right) / 2}^{1-t_{1}} \int_{1-\tau}^{t_{1}+\tau} C_{2} d t_{2} d \tau t_{1}^{\kappa-1} d t_{1}=K_{8}(\nu) B(2 \nu+1, \kappa) .
$$

22.2.4. Contribution of $C_{3}$ Notice first that

$$
\int_{1-\tau}^{t_{1}+\tau} C_{3} d t_{2}=\sum_{1 \leq k \leq \nu}\left(1-t_{1}\right)^{\nu-k}\left(1-t_{1}-\tau\right)^{\nu+k-1} \log \frac{\tau}{1-t_{1}-\tau}
$$

and then integrate aver $\tau$, getting

$$
\begin{aligned}
& \int_{\left(1-t_{1}\right) / 2}^{1-t_{1}} \int_{1-\tau}^{t_{1}+\tau} C_{3} d t_{2} d \tau \\
&=\sum_{1 \leq k \leq \nu}\left(1-t_{1}\right)^{\nu-k} \int_{\left(1-t_{1}\right) / 2}^{1-t_{1}}\left(1-t_{1}-\tau\right)^{\nu+k-1} \log \frac{\frac{\tau}{1-t_{1}}}{1-\frac{\tau}{1-t_{1}}} d \tau \\
&=\sum_{1 \leq k \leq \nu} \int_{1 / 2}^{1}(1-\tau)^{\nu+k-1} \log \frac{\tau}{1-\tau} d \tau\left(1-t_{1}\right)^{2 \nu} .
\end{aligned}
$$

Our conclusion is that there exists a coefficient $K_{9}(\nu)$ such that

$$
\int_{0}^{1} \int_{\left(1-t_{1}\right) / 2}^{1-t_{1}} \int_{1-\tau}^{t_{1}+\tau} C_{3} d t_{2} d \tau t_{1}^{\kappa-1} d t_{1}=K_{9}(\nu) B(2 \nu+1, \kappa)
$$

We again recover $K_{9}(\nu)$ by using the expression above with $t_{1}=0$ and get

$$
K_{9}(\nu)=\sum_{1 \leq k \leq \nu} \int_{1 / 2}^{1}(1-\tau)^{\nu+k-1} \log \frac{\tau}{1-\tau} d \tau=\sum_{1 \leq k \leq \nu} \frac{1}{2^{\nu+k-1}} \int_{1 / 2}^{1} \log \frac{\tau}{1-\tau} d \tau \ll 1 .
$$

22.3. When $1-\tau \leq t_{1}$ :

We use

$$
\frac{\left(1-t_{2}\right)^{\nu}-\left(1-t_{1}\right)^{\nu}}{t_{2}-t_{1}}=-\sum_{1 \leq \ell \leq \nu}\left(1-t_{2}\right)^{\nu-\ell}\left(1-t_{1}\right)^{\ell-1}
$$

We get next

$$
\begin{aligned}
& \int_{t_{1}}^{1} \sum_{1 \leq \ell \leq \nu}\left(1-t_{2}\right)^{\nu-\ell}\left(1-t_{1}\right)^{\ell-1}\left(\left(1-t_{1}\right)^{\nu}-\left(1-t_{2}\right)^{\nu}\right) d t_{2}= \\
& \sum_{1 \leq \ell \leq \nu}\left(1-t_{1}\right)^{2 \nu} \frac{1}{\nu-\ell+1}-\sum_{1 \leq \ell \leq \nu}\left(1-t_{1}\right)^{2 \nu} \frac{1}{2 \nu-\ell+1}
\end{aligned}
$$

and

$$
\begin{aligned}
\int_{1-t_{1}}^{1} \int_{t_{1}}^{1} \sum_{1 \leq \ell \leq \nu}\left(1-t_{2}\right)^{\nu-\ell}\left(1-t_{1}\right)^{\ell-1}\left(\left(1-t_{1}\right)^{\nu}-\left(1-t_{2}\right)^{\nu}\right) d t_{2} \frac{d \tau}{\tau}= \\
-\left(2 \mathcal{H}_{\nu}-\mathcal{H}_{2 \nu}\right)\left(1-t_{1}\right)^{2 \nu} \log \left(1-t_{1}\right)
\end{aligned}
$$

so that

$$
\begin{array}{r}
\int_{0}^{1} \int_{1-t_{1}}^{1} \int_{t_{1}}^{1} \sum_{1 \leq \ell \leq \nu}\left(1-t_{2}\right)^{\nu-\ell}\left(1-t_{1}\right)^{\ell-1}\left(\left(1-t_{1}\right)^{\nu}-\left(1-t_{2}\right)^{\nu}\right) d t_{2} \frac{d \tau}{\tau} t_{1}^{\kappa-1} \\
=\left(2 \mathcal{H}_{\nu}-\mathcal{H}_{2 \nu}\right) B_{1}(\kappa, 2 \nu+1)=\left(2 \mathcal{H}_{\nu}-\mathcal{H}_{2 \nu}\right)\left(\mathcal{H}_{\kappa+2 \nu}-\mathcal{H}_{2 \nu}\right) B(\kappa, 2 \nu+1) .
\end{array}
$$




\subsection{Collecting the various contibutions}

We have proved that (with $\left.w(t)=(1-t)^{+\nu}\right)$

$$
\begin{array}{r}
\int_{0}^{1} \int_{\substack{0 \leq t_{1}<t_{2} \leq 1, t_{2}-t_{1} \leq \tau}} \frac{\left(w\left(t_{2}\right)-w\left(t_{2}+\tau\right)-w\left(t_{1}\right)+w\left(t_{1}+\tau\right)\right)^{2}}{t_{2}-t_{1}} t_{1}^{\kappa-1} d t_{1} d t_{2} \frac{d \tau}{\tau} \\
=\left(K(\nu)+\left(2 \mathcal{H}_{\nu}-\mathcal{H}_{2 \nu}\right)\left(\mathcal{H}_{\kappa+2 \nu}-\mathcal{H}_{2 \nu}\right)\right) B(\kappa, 2 \nu+1) .
\end{array}
$$

with

$$
\begin{aligned}
K(\nu)=-K_{1}(\nu)+K_{2}(\nu)+K_{3}(\nu)- & K_{4}(\nu) \\
& +K_{5}-K_{6}(\nu)-K_{7}(\nu)-K_{8}(\nu)-K_{9}(\nu)+2 K_{10}(\nu) .
\end{aligned}
$$

Note that, when $\nu$ is fixed, like $\nu=1$, the dependence in $\kappa$ appears already clearly. The material above leads to an evaluation of $K(\nu)$ : we have shown that $K_{1}(\nu), K_{3}(\nu), K_{4}(\nu), K_{10}(\nu), K_{6}(\nu)$, $K_{7}(\nu), K_{8}(\nu)$ and $K_{9}(\nu)$ are all bounded by $\mathcal{O}(\log \nu)$. Furthermore, the constant $K_{2}(\nu)$ is $\frac{1}{2} \log ^{2} \nu+\mathcal{O}(\log \nu)$ so that the same evaluation holds true for $K(\nu)$.

\section{On prime $\kappa$-tuples. Proof of Theorem 1.1}

We select parameters $P=N^{\beta}$ and $Q=P$ with $2(\beta+1)<1$. Working with $Q=P^{1 / \tau_{0}}$ for some parameter $\tau_{0}$ to be chosen would in fact lead to the same bound. We set $B=\left\lceil\beta^{-1}\right\rceil$. We consider

$$
S=\sum_{1 \leq i \leq \kappa}\left(\Delta_{0} S_{i}\left(a_{0}[P]\right)+2 U S_{i}\left(a_{1}[P]\right)-U^{2}\left(2 S_{i}\left(a_{2}[P]\right)+S_{i}\left(a_{1}[P]\right)\right)\right)
$$

where $U=\vartheta / \mathcal{H}_{\kappa+2}$ for some general real parameter $\vartheta$. On recalling (1.2) and after some shuffling, we find that

$$
S=\sum_{n \leq N} \beta(n) \sum_{1 \leq i \leq \kappa}\left(\Delta_{0}+1-\left(1-U \sum_{\left\{\begin{array}{c}
p \mid n+h_{i}, \\
p \leq P
\end{array}\right.} 1\right)^{2}\right)
$$

Let us now evaluate the sum $S$ when $w(t)=(1-t)^{+}$i.e. we take $\nu=1$. By Theorem 1.2 , and because of our choice of $P$ and $Q$, the sum $S$ goes to infinity as soon as

$$
\sum_{1 \leq i \leq \kappa} \int_{0}^{1} \int_{t_{1}}^{1}\left(\Delta_{0} \mathfrak{G}_{\kappa, 0}\left(t_{1}, t_{2}, 1\right)+\left(2 U-U^{2}\right) \mathfrak{G}_{\kappa, 1}\left(t_{1}, t_{2}, 1\right)-2 U^{2} \mathfrak{G}_{\kappa, 2}\left(t_{1}, t_{2}, 1\right)\right) d t_{1} d t_{2}
$$

is at least $\eta$, for some strictly positive $\eta$ independent on $N, P$ and $Q$, but which may depend on $\kappa$. We appeal to Lemma 21.1, 21.2 and 21.3 and get that the required inequality holds when $\Delta_{0}+2 \vartheta\left(1-\vartheta\left(2 \mathcal{H}_{\kappa+2}\right)^{-1}\right)\left(1-\mathcal{H}_{\kappa+2}^{-1}\right)-\vartheta^{2}\left(1-2 \mathcal{H}_{\kappa+2}^{-1}+2 \mathfrak{B} \mathcal{H}_{\kappa+2}^{-2}\right) \geq \eta$, on writing the righthand side of Lemma 21.3 in the form $\frac{1}{2} \mathcal{H}_{\kappa+2}\left(\mathcal{H}_{\kappa+2}-2\right)+\mathfrak{B}$. Hence, the following inequality is to be fulfilled:

$$
\Delta_{0} \geq \eta-2 \vartheta+\vartheta^{2}+\frac{2 \vartheta}{\mathcal{H}_{\kappa+2}}+\frac{\vartheta^{2}(\mathfrak{B}-1)}{\mathcal{H}_{\kappa+2}^{2}} .
$$

We select $\eta=1 / \mathcal{H}_{\kappa+2}^{2}$ and

$$
\Delta_{0}=-2 \vartheta+\vartheta^{2}+\frac{2 \vartheta}{\mathcal{H}_{\kappa+2}}+\frac{1+\vartheta^{2}(\mathfrak{B}-1)}{\mathcal{H}_{\kappa+2}^{2}} .
$$


We denote by $\infty(N)$ a function that goes to infinity with $N$. With the choice $(23.2)$, there are $\infty(N)$ many integers $n$ such that

$$
\kappa\left(\Delta_{0}+1\right) \mathcal{H}_{\kappa+2}^{2}-\sum_{1 \leq i \leq \kappa}\left(\mathcal{H}_{\kappa+2}-\vartheta \omega\left(n+h_{i} ; P\right)\right)^{2}>0
$$

where $\omega(m ; P)$ denotes the number of prime factors of $m$ that are not more than $P$ counted without multiplicity. The quantity $1+\Delta_{0}$ goes to zero only with the choice $\vartheta=1$. We continue with this choice. We replace $\mathcal{H}_{\kappa+2}$ by $\log \kappa$ as follows. Put $\Theta_{\kappa}=\mathcal{H}_{\kappa+2}-\log \kappa=\mathcal{O}(1)$. We expand the quantity above as

$$
\sum_{1 \leq i \leq \kappa}\left[\left(\log \kappa-\omega\left(n+h_{i} ; P\right)\right)^{2}+2\left(\mathcal{H}_{\kappa+2}-\omega\left(n+h_{i} ; P\right)\right) \Theta_{\kappa}-\Theta_{\kappa}^{2}\right]
$$

We treat the second sum by using Cauchy. Our last task is to replace $\omega(m ; P)$ by $\omega(m)$. We first notice that an integer $m \leq N$ can have at most $B$ prime factors $>P$, which implies that $\omega(m) \geq \omega(m ; P) \geq \omega(m)-B$. We set $E_{\kappa}(m ; P)=\omega(m)-\omega(m ; P)$ and use the decomposition:

$$
\sum_{1 \leq i \leq \kappa}\left[\left(\log \kappa-\omega\left(n+h_{i}\right)\right)^{2}+2\left(\mathcal{H}_{\kappa+2}-\omega\left(n+h_{i}\right)\right) E_{\kappa}\left(n+h_{i} ; P\right)+E_{\kappa}\left(n+h_{i} ; P\right)^{2}\right] \text {. }
$$

We again treat the second sum by using Cauchy. We finally select $P=Q=N^{2 / 9}$ and the Theorem 1.1.

\section{References}

1. M. Abramowitz and I.A. Stegun. Handbook of mathematical functions, volume 55 of Applied Mathematics Series. National Bureau of Standards, 1964. http://mintaka.sdsu.edu/faculty/wfw/ABRAMOWITZ-STEGUN.

2. E. Bombieri. Le grand crible dans la théorie analytique des nombres. Astérisque, 18:103pp, 1987/1974.

3. L. Brandolini, L. Colzani, and G. Travaglini. Average decay of Fourier transforms and integer points in polyhedra. Arkiv för Matematik, 35(2):253-275, 1997.

4. V. Brun. Le crible d'Erathosthène et le théorème de Goldbach. C.R., 168:544-546, 1919.

5. H.G. Diamond, H. Halberstam, and W.F. Galway. A higher-dimensional sieve method, volume 177 of Cambridge tracts in mathematics. Cambridge University Press, 2008.

6. P.X. Gallagher. Sieving by prime powers. Acta Arith., 24:491-497, 1974.

7. D.A. Goldston, S.W. Graham, J. Pintz, and C.Y. Yıldırım. Small gaps between products of two primes. Proc. London Math. Soc., 98(3):741-774, 2009.

8. G. Greaves. Sieves in number theory, volume 43 of Ergebnisse der Mathematik und ihrer Grenzgebiete. Springer-Verlag, Berlin, 2001. xii+304 pp.

9. H. Halberstam and H.E. Richert. Mean value theorems for a class of arithmetic functions. Acta Arith. 43:243-256, 1971.

10. H. Halberstam and H.E. Richert. Sieve methods. Academic Press (London), page 364pp, 1974.

11. G.H. Hardy and J.E. Littlewood. Some problems of "Partitio Numerorum" III. On the expression of a number as a sum of primes. Acta Math., 44:1-70, 1922.

12. D.R. Heath-Brown. Almost-prime $k$-tuples. Mathematika, 44(2):245-266, 1997.

13. I. Kobayashi. A note on the Selberg sieve and the large sieve. Proc. Japan Acad., 49(1):1-5, 1973

14. P. Kuhn. Zur Viggo Brun'schen Siebmethode. I. Norske Vid. Selsk. Forhdl., Trondheim, 14:145-148, 1941

15. Maxima.sourceforge.net. Maxima, a Computer Algebra System. Technical Report Version 5.21.1, 2010. http://maxima. sourceforge.net/.

16. R.J. Miech. Almost primes generated by a polynomial. Acta Arith., 10:9-30, 1964.

17. Y. Motohashi. Primes in arithmetic progressions. Invent. Math., 44(2):163-178, 1978.

18. D.S. Ramana. Averaged decay rate of the fourier transforms of piecewise affine linear functions on $\mathbb{R}^{n}$, personal communication. 2010.

19. O. Ramaré. Arithmetical aspects of the large sieve inequality, volume 1 of Harish-Chandra Research Institute Lecture Notes. Hindustan Book Agency, New Delhi, 2009. With the collaboration of D. S. Ramana.

20. O. Ramaré and I.M. Ruzsa. Additive properties of dense subsets of sifted sequences. J. Théorie $N$. Bordeaux, 13:559-581, 2001.

21. S. Salerno. A note on Selberg sieve. Acta Arith., 45(4):279-288, 1986

22. L. Schwartz. Analyse IV. Collection Enseignement des Sciences, Hermann, 1993.

23. A. Selberg. Remarks on multiplicative functions. Lectures Notes in Mathematics (Berlin), 626:232-241, 1976. 
24. A. Selberg. Sifting problems, sifting density, and sieves. In D. Goldfeld" "K.E. Aubert, E. Bombieri, editor, Number Theory, Trace Formulas and Discrete Groups, pages 467-484, Oslo, 1987. Academic Press, San Diego London.

25. M. Tarnopolska-Weiss. On the number of lattice points in a compact $n$-dimensional polyhedron. Proceedings of the American Mathematical Society, 74(1):124-127, 1979.

26. J.E. van Lint and H.E. Richert. On primes in arithmetic progressions. Acta Arith., 11:209-216, 1965.

27. Wxmaxima.sourceforge.net. wxMaxima, a GUI for the Maxima System. Technical Report Version 0.8.5, 2010. http://wxmaxima. sourceforge.net/.

\section{Olivier Ramaré}

CNRS / Laboratoire Paul Painlevé,

Université Lille 1,

59655 Villeneuve d'Ascq, cedex,

France.

ramare@math.univ-lille1.fr 